\title{
HIGHER ORDER LOCAL DIRICHLET INTEGRALS AND DE BRANGES-ROVNYAK SPACES
}

\author{
SHUAIBING LUO, CAIXING GU, AND STEFAN RICHTER
}

\begin{abstract}
We investigate expansive Hilbert space operators $T$ that are finite rank perturbations of isometric operators. If the spectrum of $T$ is contained in the closed unit disc $\overline{\mathbb{D}}$, then such operators are of the form $T=U \oplus R$, where $U$ is isometric and $R$ is unitarily equivalent to the operator of multiplication by the variable $z$ on a de Branges-Rovnyak space $\mathcal{H}(B)$. In fact, the space $\mathcal{H}(B)$ is defined in terms of a rational operator-valued Schur function $B$. In the case when $\operatorname{dim} \operatorname{ker} T^{*}=1$, then $\mathcal{H}(B)$ can be taken to be a space of scalar-valued analytic functions in $\mathbb{D}$, and the function $B$ has a mate $a$ defined by $|B|^{2}+|a|^{2}=1$ a.e. on $\partial \mathbb{D}$. We show the mate $a$ of a rational $B$ is of the form $a(z)=a(0) \frac{p(z)}{q(z)}$, where $p$ and $q$ are appropriately derived from the characteristic polynomials of two associated operators. If $T$ is a $2 m$-isometric expansive operator, then all zeros of $p$ lie in the unit circle, and we completely describe the spaces $\mathcal{H}(B)$ by use of what we call the local Dirichlet integral of order $m$ at the point $w \in \partial \mathbb{D}$.
\end{abstract}

Keywords: De Branges-Rovnyak space; $m$-isometry.

\section{Contents}

1. Introduction

2. Preliminaries

3. Finite rank extensions

4. De Branges-Rovnyak spaces and expansive operators

5. Some observations about Schur functions with $B(0)=0$.

6. Rational matrix-valued Schur class functions

7. Rational Row Schur functions

8. Expansive $m$-isometries, general considerations

9. Expansive $2 m$-isometries, rank $\Delta=1$

10. Finite rank expansive $2 m$-isometries

11. A Construction

References

Date: September 1, 2020.

2010 Mathematics Subject Classification. 47B38, 46E22, 47A45, 47A67.

S. Luo was supported by NNSFC (\# 11701167). 


\section{INTRODUCTION}

Let $\mathbb{D}$ be the open unit disc in the complex plane $\mathbb{C}$, and $\mathbb{T}=\partial \mathbb{D}$ be the unit circle. If $b: \mathbb{D} \rightarrow \mathbb{D}$ is analytic, then the de Branges-Rovnyak space $\mathcal{H}(b)$ is the unique Hilbert space of holomorphic functions on $\mathbb{D}$ with reproducing kernel

$$
K_{w}^{b}(z)=\frac{1-b(z) \overline{b(w)}}{1-z \bar{w}}
$$

i.e. $K^{b}$ satisfies $f(w)=\left\langle f, K_{w}^{b}\right\rangle$ for all $f \in \mathcal{H}(b)$. De Branges-Rovnyak spaces possess a rich structure, and they play an important role in many aspects of complex analysis and operator theory. We refer the reader to the books of de Branges and Rovnyak [19], Sarason [38], Fricain and Mashreghi [23], and for some recent developments to [7, 11-13, 15-18, $21,22,27,28,30$, and the references therein.

It is well-known that the backward shift $L f(z)=\frac{f(z)-f(0)}{z}$ acts contractively on every de Branges-Rovnyak space, but the forward shift $\left(M_{z}, \mathcal{H}(b)\right)$ defined by $\left(M_{z} f\right)(z)=z f(z)$ is bounded only if $b$ is not an extreme point of the unit ball of $H^{\infty}$, see [38]. This condition is known to be equivalent to the existence of a mate for $b$, i.e. an outer function $a$ such that $|a|^{2}+|b|^{2}=1$ a.e. on the unit circle $\mathbb{T}$. The mate is unique, if we also assume that $a(0)>0$. In this paper we will make this assumption, and then refer to the unique mate as the mate of $b$.

Thus, if such a forward shift is bounded, then it expands the norm. In this paper we investigate which expansive operators $T$ are unitarily equivalent to $\left(M_{z}, \mathcal{H}(b)\right)$ for rational functions $b$, and we obtain further results that link properties of $T$ and of $b$. It will follow from Lemma 4.7 that, if for $\alpha \in \mathbb{D} b_{\alpha}(z)=\frac{\alpha-b(z)}{1-\bar{\alpha} b(z)}$, then $\left(M_{z}, \mathcal{H}(b)\right)$ is unitarily equivalent to $\left(M_{z}, \mathcal{H}\left(b_{\alpha}\right)\right)$. Hence there will be no loss of generality in assuming that $b(0)=0$.

If $b$ is a rational function, then the degree of $b$ is defined to be the larger of the degrees of the polynomials $p$ and $q$ provided that $b=\frac{p}{q}$ is in reduced form. In [39] Sarason observed that if $b$ is a certain rational function of degree 1 , then $\mathcal{H}(b)$ equals a so-called local Dirichlet space. Local Dirichlet spaces are important for the study of two-isometric operators, i.e. bounded linear operators $T \in \mathcal{B}(\mathcal{H})$ that satisfy $T^{* 2} T^{2}-$ $2 T^{*} T+I=0$, see e.g. 3234 . Sarason's result has been refined and extended in [16, 17, 20, 27], and one of their results can be paraphrased as follows: If $b(0)=0$, then $\left(M_{z}, \mathcal{H}(b)\right)$ is a two-isometry, if and only if

$$
b(z)=e^{i t} \frac{(1-r) \bar{w} z}{1-r \bar{w} z}
$$


for some $t \in[0,2 \pi), 0<r \leq 1$ and $|w|=1$. In this case $\|f\|_{\mathcal{H}(b)}^{2}=$ $\|f\|_{H^{2}}^{2}+\frac{(1-r)^{2}}{r} D_{w}(f)$, where $D_{w}(f)=\int_{|z|=1}\left|\frac{f(z)-f(w)}{z-w}\right|^{2} \frac{|d z|}{2 \pi}$ is the local Dirichlet integral of $f$, see Theorem 3.1 of [16].

Let $m \in \mathbb{N}$. An operator $T \in \mathcal{B}(\mathcal{H})$ is called an $m$-isometry, if

$$
\beta_{m}(T)=\sum_{k=0}^{m}(-1)^{m-k}\left(\begin{array}{c}
m \\
k
\end{array}\right) T^{* k} T^{k}=0 .
$$

The study of $m$-isometries originated in the work of Agler [1, Condition (2.7), and it was at least partially motivated by an analogy with Helton's study of $m$-symmetric operators, [26]. The first in-depth study of $m$-isometries was carried out in [4], and by now there is an extensive list of references for these operators.

Since $\beta_{m+1}(T)=T^{*} \beta_{m}(T) T-\beta_{m}(T)$ it follows that every $m$-isometry is a $k$-isometry for every $k \geq m$, and we say that $T$ is a strict $m$ isometry, if $\beta_{m-1}(T) \neq 0$. If $m \in \mathbb{N}, w \in \mathbb{T}$, and if $f \in H^{2}$ extends to be analytic in a neighborhood of $w$, then we define the local Dirichlet integral of $f$ of order $m$ at $w$ by

$$
D_{w}^{m}(f)=\int_{|z|=1}\left|\frac{f(z)-T_{m-1}(f, w)(z)}{(z-w)^{m}}\right|^{2} \frac{|d z|}{2 \pi},
$$

where $T_{m-1}(f, w)$ is the $(m-1)$-th order Taylor polynomial of $f$ at $w$. This definition can be extended to apply to more general functions, see Section 9. In particular, we note that $D_{w}^{1}(f)=D_{w}(f)$ for all $f$. The space $\mathcal{D}_{w}^{m}$ is defined to consist of all $f \in H^{2}$ such that $D_{w}^{m}(f)<\infty$. We will show

Theorem 1.1. Let $b$ be a non-extreme point of the unit ball of $H^{\infty}$ with $b(0)=0$, and let $m \in \mathbb{N}$. Then $\left(M_{z}, \mathcal{H}(b)\right)$ is not a strict $(2 m+1)$ isometry, and the following are equivalent:

(i) $\left(M_{z}, \mathcal{H}(b)\right)$ is a strict $2 m$-isometry,

(ii) $b$ is a rational function of degree $m$ such that the mate has a single zero of multiplicity $m$ at a point $w \in \mathbb{T}$,

(iii) there is a $w \in \mathbb{T}$ and a polynomial $\tilde{p}$ of degree $<m$ with $\tilde{p}(w) \neq$ 0 such that $\|f\|_{\mathcal{H}(b)}^{2}=\|f\|_{H^{2}}^{2}+D_{w}^{m}(\tilde{p} f)$.

If the three conditions hold, then there are polynomials $p$ and $q$ of degree $\leq m$ such that $b=\frac{p}{q}, a=\frac{(z-w)^{m}}{q}, \tilde{p}(z)=z^{m} \overline{p(1 / \bar{z})}$ for $z \in \mathbb{D}$, and $|q(z)|^{2}=|p(z)|^{2}+|z-w|^{2 m}$ for all $z \in \mathbb{T}$. Furthermore, $\mathcal{H}(b)=\mathcal{D}_{w}^{m}$ with equivalence of norms.

Note that if $m=1$ in this theorem, then $|b(w)|=1$ for some $w \in \mathbb{T}$, and then the conditions that $b(0)=0$ and that $b$ has degree 1 imply 
that it has the form as in (1.1) for $r<1$. And in fact, one checks that for two-isometries this theorem is equivalent to the earlier one.

Theorem 1.1 raises two questions:

1. If $b$ is a more general rational function and non-extreme, then what operator properties of the forward and backward shifts on $\mathcal{H}(b)$ can be easily seen by looking at the mate $a$ of $b$ ?

2. Among all $m$-isometries, how general a class are the ones that are unitarily equivalent to $\left(M_{z}, \mathcal{H}(b)\right)$ for some non-extreme $b$ ?

We give answers to both questions. In fact, it will be instructive to consider the vector-valued de Branges-Rovnyak spaces $\mathcal{H}(B)$, where $B \in \mathcal{S}(\mathcal{D}, \mathcal{E})$ is an operator-valued Schur class function, i.e. $B: \mathbb{D} \rightarrow \mathcal{B}(\mathcal{D}, \mathcal{E})$ is a contractive analytic function. Our results are most complete in the interesting case where $H(B)$ is a space of scalarvalued analytic functions, i.e. when $\mathcal{E}=\mathbb{C}$.

An operator $T$ is called analytic, if $\bigcap_{n \geq 0} \operatorname{ran} T^{n}=(0)$. It turns out that by combining a construction of Shimorin's ( [37]) and theorems of Aleman and Malman [7] one sees rather easily that a Hilbert space operator $T$ is norm expansive and analytic if and only if it is unitarily equivalent to $\left(M_{z}, \mathcal{H}(B)\right)$ for some operator-valued Schur class function $B$ with $B(0)=0$. We will need details from this construction, thus we have included the complete details in Section 4 .

For $T \in \mathcal{B}(\mathcal{H})$ write $\Delta=T^{*} T-I$. In particular, we will see that an operator $T$ is unitarily equivalent to $\left(M_{z}, \mathcal{H}(b)\right)$ for some non-extremal $b$ in the unit ball of $H^{\infty}$, if and only if $T$ is analytic, $\operatorname{dim} \operatorname{ker} T^{*}=1$, and $\Delta$ is positive and has rank 1 . In the more general case where $T$ is analytic, $\operatorname{dim} \operatorname{ker} T^{*}=1$ and $\Delta \geq 0$ one can take $B=\left(b_{1}, b_{2}, \ldots\right)$ for scalar-valued analytic functions $b_{j}$ and one obtains a space $\mathcal{H}(B)$ of scalar-valued analytic functions with reproducing kernel $K_{w}^{B}(z)=$ $\frac{1-\sum_{i=1}^{\infty} b_{i}(z) \overline{b_{i}(w)}}{1-z \bar{w}}$. The minimum number of functions $b_{j}$ that are not identically equal to 0 equals the rank of $\Delta$, see the beginning of Section 7. As before, the condition $B(0)=0$ can be seen as a normalization that assures that $\mathcal{H}(B)$ contains the constant functions. This set-up applies for example to $T=\sqrt{2}\left(M_{z}, L_{a}^{2}\right)$, where $\left(M_{z}, L_{a}^{2}\right)$ is the forward shift on the Bergman space of the unit disc. Of course, in this case $\mathcal{H}(B)$ will be a space of analytic functions on a disc of radius $\sqrt{2}$, and one may not expect to easily obtain deep information by looking at the corresponding Schur function $B$. However, for many interesting expansive operators $T$ the defect operator $\Delta=T^{*} T-I$ will be compact. That is true for example for the Dirichlet shift, and more generally, if $T=M_{z}$ on a superharmonically weighted Dirichlet space (see [29], Theorem 5.1). If this is the case, then $\Delta=\sum_{n \geq 1} t_{n} f_{n} \otimes f_{n}$ for some 
$0<t_{n} \rightarrow 0$ and an orthonormal basis $\left\{f_{n}\right\}$ of $\overline{\operatorname{ran} \Delta}$, and one can show that for the $B$ one can take $b_{n} / z=\sqrt{\frac{t_{n}}{1+t_{n}}} f_{n}$. This follows from the proofs of Lemmas 4.5 and 5.1 .

In this paper we will be interested in the situation, where $\Delta$ is a finite rank operator. For that case we can take $B=\left(b_{1}, \ldots, b_{k}\right)$ and Aleman and Malman proved that $M_{z}$ acts boundedly on $\mathcal{H}(B)$, if and only if $1-\sum_{i=1}^{k}\left|b_{i}\right|^{2}$ is log-integrable on $\mathbb{T}$, [7]. Thus, as in the classical rank 1 case such $B$ will have a mate $a$, the unique outer function with $a(0)>0$ and $|a|^{2}+\sum_{i=1}^{k}\left|b_{i}\right|^{2}=1$ a.e. on $\mathbb{T}$.

Crucial to all our results will be the space $\mathcal{N}=[\operatorname{ran} \Delta]_{T^{*}}$, the smallest $T^{*}$-invariant subspace that contains ran $\Delta$. It is not difficult to show that $\mathcal{M}=\mathcal{N}^{\perp}$ is the largest $T$-invariant subspace such that $T \mid \mathcal{M}$ is isometric. We will show that $\mathcal{N}=[\operatorname{ran} \Delta]_{L}$, the smallest $L$-invariant subspace that contains ran $\Delta$, see Lemma 6.1. Here $L f(z)=\frac{f(z)-f(0)}{z}$ is the backward shift. Of course, this space may be all of $\mathcal{H}(B)$. Our theorem describes a case when this does not happen. $B$ is called rational, if each $b_{i}$ is a rational function. By taking common denominators it follows that rational $B$ are of the form $B=\left(p_{1} / q, \ldots, p_{k} / q\right)$, where $q, p_{1}, \ldots p_{k}$ are polynomials. The degree of $B$ is defined to be the smallest $n$ such that $B$ has such a representation where the degrees of all polynomials are less than or equal to $n$. So for example, $B(z)=\left(\frac{1}{5+z} \frac{1}{6+z}\right)$ has degree 2 . Recall that the characteristic polynomial $p$ of an $n \times n$ matrix $A$ is defined by $p(z)=\operatorname{det}(z I-A)$.

Theorem 1.2. Let $B=\left(b_{1}, \ldots, b_{k}\right)$ be such that $B(0)=0$ and $1-$ $\sum_{i=1}^{k}\left|b_{i}\right|^{2}$ is log-integrable on $\mathbb{T}$. Let $T=\left(M_{z}, \mathcal{H}(B)\right)$ and $\Delta=T^{*} T-I$.

Then $B$ is a rational function of degree $n$, if and only if

$$
\operatorname{dim}[\operatorname{ran} \Delta]_{T^{*}}=n<\infty .
$$

Furthermore, if $\mathcal{N}=[\text { ran } \Delta]_{T^{*}}$ has dimension $n<\infty$, and if $p(z)=$ $\prod_{i=1}^{n}\left(z-w_{i}\right)$ is the characteristic polynomial of $T^{*} \mid \mathcal{N}$ and $q(z)=$ $\prod_{i=1}^{n}\left(z-\alpha_{i}\right)$ is the characteristic polynomial of $L \mid \mathcal{N}$, then

$$
a(z)=a(0) \frac{\prod_{i=1}^{n}\left(1-w_{i} z\right)}{\prod_{i=1}^{n}\left(1-\alpha_{i} z\right)} .
$$

We approach the second question by first stating a theorem for more general expansive operators with finite rank defect $\Delta$.

Theorem 1.3. Let $T \in \mathcal{B}(\mathcal{H})$ with $\Delta=T^{*} T-I \geq 0$, and let $\mathcal{N}=$ $[\operatorname{ran} \Delta]_{T^{*}}$.

Then the following are equivalent:

(a) $\mathcal{N}$ is finite dimensional and $\sigma(T) \subseteq \overline{\mathbb{D}}$, 
(b) $T=V \oplus R$, where $V$ is isometric and $R$ is unitarily equivalent to $\left(M_{z}, \mathcal{H}(B)\right)$ for some rational $B \in \mathcal{S}\left(\mathbb{C}^{k}, \mathbb{C}^{m}\right)$.

If (a) and (b) are satisfied, then one can choose $k=\operatorname{dim} \operatorname{ran} \Delta$, $m=\operatorname{dim} \operatorname{ran}\left(I-P_{\mathcal{N}}\right) T P_{\mathcal{N}}=\operatorname{dim} \operatorname{ker} R^{*}$, and one has

$$
\text { degree } B \leq \operatorname{dim} \mathcal{N} \leq m \text { degree } B
$$

The degree of a rational matrix-valued function $B(z)=\left(b_{i j}(z)\right)_{i j}$ is defined analogously to the way it was defined for row operator-valued functions.

Two-isometric operators are automatically norm expansive. That is no longer true for $m$-isometries, if $m \geq 3$. For example, one easily checks that $T=\left[\begin{array}{cc}1 & \alpha \\ 0 & 1\end{array}\right], \alpha \neq 0$ defines a 3 -isometric operator on $\mathbb{C}^{2}$ that is not norm expansive. Still, for each $m \in \mathbb{N}$ there are examples of norm expansive strict $m$-isometries, see e.g. [25]. For norm expansive $m$ isometries with finite rank defect operator $\Delta$ there are some restrictions.

Theorem 1.4. Let $T \in \mathcal{B}(\mathcal{H})$ be such that $\Delta=T^{*} T-I$ is a positive operator of finite rank, and let $m \in \mathbb{N}$.

(a) If $T$ is a $2 m+1$-isometry, then $T$ is a $2 m$-isometry.

(b) $T$ is a $2 m$-isometry, if and only if there are $w_{1}, \ldots, w_{k} \in \mathbb{T}$ and positive operators $\Delta_{1}, \ldots, \Delta_{k}$ such that $\Delta=\sum_{j=1}^{k} \Delta_{j}$ and $\left(T^{*}-\right.$ $\left.\bar{w}_{j}\right)^{m} \Delta_{j}=0$ for each $j$.

Furthermore, if the above is satisfied with $\Delta_{j} \neq 0$ for all $j$ and if $\mathcal{N}=$ $[\operatorname{ran} \Delta]_{T^{*}}$, then $\mathcal{N}$ is finite dimensional and $\sigma\left(T^{*} \mid \mathcal{N}\right)=\left\{\bar{w}_{1}, \ldots, \bar{w}_{k}\right\}$.

Theorem 1.3 implies that if one wants to classify the expanding $2 \mathrm{~m}$ isometries with finite rank $\Delta$, then one needs to understand the $2 \mathrm{~m}$ isometries acting on de Branges-Rovnyak spaces $\mathcal{H}(B)$. It turns out that if $B \in \mathcal{S}\left(\mathbb{C}^{n}, \mathbb{C}\right)$, then $T=\left(M_{z}, \mathcal{H}(B)\right)$ is a $2 m$-isometry, if and only if $\mathcal{H}(B)$ can be represented as an intersection of finitely many rank 1 spaces $H\left(b_{i}\right)$ as considered in Theorem 1.1 .

Theorem 1.5. Let $B \in \mathcal{S}\left(\mathbb{C}^{N}, \mathbb{C}\right)$ be a contractive analytic function with $B(0)=0$ and such that $T=\left(M_{z}, \mathcal{H}(B)\right)$ is bounded and satisfies rank $\Delta<\infty$. Let $\mathcal{N}=[\operatorname{ran} \Delta]_{T^{*}}$.

Then $T$ is a $2 m$-isometry, if and only if there are $w_{1}, \ldots, w_{k} \in \mathbb{T}$, pairs of integers $\left(m_{1}, n_{1}\right), \ldots\left(m_{k}, n_{k}\right)$ such that $1 \leq n_{j} \leq m_{j} \leq m$ for all $j$, and there are polynomials $\left\{p_{i j}\right\}_{1 \leq j \leq k, 1 \leq i \leq n_{j}}$ with degree $p_{i j} \leq$ $m_{j}-1$ for $1 \leq j \leq k, 1 \leq i \leq n_{j}$ such that 


$$
\|f\|_{\mathcal{H}(B)}^{2}=\|f\|_{H^{2}}^{2}+\sum_{j=1}^{k} \sum_{i=1}^{n_{j}} D_{w_{j}}^{m_{j}}\left(p_{i j} f\right) .
$$

There is a choice of parameters so that for each $j$ there is an $i$ with $p_{i j}\left(w_{j}\right) \neq 0$ and such that $\sum_{j=1}^{k} n_{j}=$ rank $\Delta$. If all that is the case, then $\mathcal{H}(B)=\bigcap_{j=1}^{k} \mathcal{D}_{w_{j}}^{m_{j}}$ with equivalence of norms, the characteristic polynomial of $A=P_{\mathcal{N}} T \mid \mathcal{N}$ is $p_{A}(z)=\prod_{j=1}^{k}\left(z-w_{j}\right)^{m_{j}}$, and the mate $a$ of $B$ is of the form $a(z)=\frac{p_{A}(z)}{q(z)}$, where $q$ is the unique polynomial of degree $\leq \sum_{j=1}^{k} m_{j}$, which has no zeros in $\overline{\mathbb{D}}$, and satisfies $\frac{p_{A}(0)}{q(0)}>0$ and

$$
|q(z)|^{2}=\left|p_{A}(z)\right|^{2}+\sum_{j=1}^{k}\left|\frac{p_{A}(z)}{\left(z-w_{j}\right)^{m_{j}}}\right|^{2} \sum_{i=1}^{n_{j}}\left|p_{i j}(z)\right|^{2} \quad \text { for all }|z|=1 .
$$

In the scalar and rank 1 case a formula for the norm of a function $f \in \mathcal{H}(b)$ is given by $\|f\|_{\mathcal{H}(b)}^{2}=\|f\|_{H^{2}}^{2}+\left\|f^{+}\right\|_{H^{2}}^{2}$, where $f^{+}$is a function that is appropriately associated with $f$, see [38], section IV-1. Formula (1.2) is the analogue of this for the case considered in the theorem. An important ingredient to derive (1.2) is a formula of Aleman-Malman, see Lemma 10.1 .

In the above we mentioned the following useful boundedness criterion of Aleman and Malman.

Theorem 1.6. ( [7], Theorem 5.2) If $B=\left(b_{1}, \ldots, b_{n}\right) \in \mathcal{S}\left(\mathbb{C}^{n}, \mathbb{C}\right)$, then $\left(M_{z}, \mathcal{H}(B)\right)$ is bounded, if and only if

$$
\int_{|z|=1} \log \left(1-\sum_{i=1}^{n}\left|b_{i}(z)\right|^{2}\right) \frac{|d z|}{2 \pi}>-\infty .
$$

Simple examples show that this condition does not capture the complete characterization for boundedness in the general case, see Example 4.4. It is thus worthwhile to point out that routine methods establish the following necessary and sufficient condition, see Lemma 4.3 .

Theorem 1.7. Let $B \in \mathcal{S}(\mathcal{D}, \mathcal{E})$ with $B(0)=0$, then the following are equivalent:

(i) $T: f \rightarrow z f$ defines a bounded operator on $\mathcal{H}(B)$,

(ii) for each $x \in \mathcal{D}$ the function $g_{x}(z)=B(z) x \in \mathcal{H}(B)$.

The paper is organized as follows. In Section 3 we consider general Hilbert space operators $T \in \mathcal{B}(\mathcal{H})$ such that $\Delta=T^{*} T-I \geq 0$ and that have the additional property that $\mathcal{N}=[\operatorname{ran} \Delta]_{T^{*}}$ is finite dimensional. 
In Theorem 3.4 we will establish the implication $(\mathrm{a}) \Rightarrow(\mathrm{b})$ of Theorem 1.3. The reverse implication and the rest of Theorem 1.3 will follow from Theorem 6.2. In Section 4 we present our approach to the fact that all expansive analytic operators can be represented as $M_{z}$ on a de Branges-Rovnyak space $\mathcal{H}(B)$ for some operator-valued Schur function $B$, see Theorem 4.6. The theorem says that one may always assume that $B(0)=0$. If $B$ and $C$ are two scalar-valued Schur functions with $B(0)=C(0)=0$, then it turns out that the operators $\left(M_{z}, \mathcal{H}(B)\right)$ and $\left(M_{z}, \mathcal{H}(C)\right)$ are unitarily equivalent, if and only if $\mathcal{H}(B)=\mathcal{H}(C)$ with equality of norms, see Lemma 4.8. Section 5 contains some background facts about general Schur functions with $B(0)=0$. We already mentioned that in Theorem 6.2 we will establish the remaining parts of Theorem 1.3. Additionally, we show that any rational Schur function $B$ is of the form $B(z)=\frac{1}{\tilde{q}(z)} P(z)$ for some operator polynomial $P$ and the scalar function $\tilde{q}(z)=z^{n} q(1 / z)$, where $q$ is the characteristic polynomial of $L \mid \mathcal{N}$ and $n=\operatorname{dim} \mathcal{N}$. In Section 7 we restrict attention to scalar-valued Schur functions, and Theorem 1.2 will follow from Theorems 6.2 and 7.2. In Sections 8-10 we prove our theorems about expansive $n$-isometric operators. The proof of Theorem 8.2 contains an elementary argument that will establish one of the implications in part (b) of Theorem 1.4. The main ingredient for the remainder of Theorem 1.4 will be a construction in which we put a new norm on $\mathcal{N}$ in such a way that it allows us to use results of Agler-Helton-Stankus ( [5]) about $n$-isometries on finite dimensional spaces. In Section 9 we give the rigorous definition of the higher order local Dirichlet integral and we prove some general facts about the spaces $\mathcal{D}_{w}^{m}$. We also establish Theorem 9.4, which is a version of Theorem 1.1. Corollary 9.5 says that for a fixed $w \in \mathbb{T}$ all spaces $\mathcal{H}(b)$ as defined by (iii) of Theorem 1.1 equal $\mathcal{D}_{w}^{m}$ with equivalence of norms. In Section 10 we present the proof of Theorem 1.5, see Theorems 10.4 and 10.5. Finally, in Section 11 we outline method of how to calculate the Schur function $B$ in Theorem 1.5, if one knows the polynomials $p_{i j}$ and the rank of $\Delta$.

\section{PRELIMINARIES}

We start with a lemma that is probably well-known, we include the proof for completeness. Recall from the Introduction that $T \in$ $\mathcal{B}(\mathcal{H})$ is called analytic, if $\bigcap_{n \geq 1} \operatorname{ran} T^{n}=(0)$. This is equivalent to $\bigvee_{n \geq 1} \operatorname{ker} T^{* n}=\mathcal{H}$. 
Lemma 2.1. Let $0<\varepsilon<1$ and let $T \in \mathcal{B}(\mathcal{H})$ with $\|T x\| \geq\|x\|$ for all $x \in \mathcal{H}$. Then

$$
\bigvee_{n \geq 1} \operatorname{ker} T^{* n}=\bigvee_{|\lambda|<\varepsilon} \operatorname{ker}\left(T^{*}-\bar{\lambda}\right)
$$

Proof. Since $T$ is bounded below, the operator $T^{*} T$ is invertible. We set $L=\left(T^{*} T\right)^{-1} T^{*}$, the left inverse of $T$ with $\operatorname{ker} T^{*}=\operatorname{ker} L$. Note that $T L$ is a projection, thus $\|L x\| \leq\|T L x\| \leq\|x\|$. Hence $\left(1-\bar{\lambda} L^{*}\right)^{-1}$ exists for all $|\lambda|<1$.

Fix $|\lambda|<\varepsilon$ and let $x \in \operatorname{ker}\left(T^{*}-\bar{\lambda}\right)$. Set $y=\left(1-\bar{\lambda} L^{*}\right) x$, and observe that $y \in \operatorname{ker} T^{*}$ since $T^{*} L^{*}=I$. For $N \in \mathbb{N}$ let $x_{N}=\sum_{k=0}^{N} \bar{\lambda}^{k} L^{* k} y$, then $T^{* N+1} x_{N}=\sum_{k=0}^{N} \bar{\lambda}^{k} T^{* N+1-k} y=0$. Thus $x_{N} \in \bigvee_{n \geq 1} \operatorname{ker} T^{* n}$ and $x_{N} \rightarrow\left(1-\bar{\lambda} L^{*}\right)^{-1} y=x$. This implies that

$$
\bigvee_{|\lambda|<\varepsilon} \operatorname{ker}\left(T^{*}-\bar{\lambda}\right) \subseteq \bigvee_{n \geq 1} \operatorname{ker} T^{* n}
$$

In order to show the reverse inclusion, we set $\mathcal{M}=\bigvee_{|\lambda|<\varepsilon} \operatorname{ker}\left(T^{*}-\bar{\lambda}\right)$, and we will start by showing that $\mathcal{M}$ is invariant for $L^{*}$. First we let $0<|\lambda|<\varepsilon$ and $x \in \operatorname{ker}\left(T^{*}-\bar{\lambda}\right)$. Then

$$
L^{*} x=\frac{1}{\bar{\lambda}} x-\frac{1}{\bar{\lambda}}\left(1-\bar{\lambda} L^{*}\right) x
$$

is a difference of an element in $\operatorname{ker}\left(T^{*}-\bar{\lambda}\right)$ and an element in $\operatorname{ker} T^{*}$. Thus, $L^{*} x \in \mathcal{M}$ whenever $x \in \operatorname{ker}\left(T^{*}-\bar{\lambda}\right)$ for $\lambda \neq 0$. If $x \in \operatorname{ker} T^{*}$, then for small $|\lambda| \neq 0$ we have

$$
y_{\lambda}=\left(1-\bar{\lambda} L^{*}\right)^{-1} x \in \operatorname{ker}\left(T^{*}-\bar{\lambda}\right) .
$$

Then $L^{*} y_{\lambda} \in \mathcal{M}$, and $L^{*} x \in \mathcal{M}$ follows from the fact that $L^{*} y_{\lambda} \rightarrow L^{*} x$ as $|\lambda| \rightarrow 0$. Thus, $\mathcal{M}$ is $L^{*}$-invariant.

We will now use induction on $n$ to show that $\operatorname{ker} T^{* n} \subseteq \mathcal{M}$ for each $n \geq 1$. The conclusion is obvious, if $n=1$. Suppose that $n \geq 1$ and that $\operatorname{ker} T^{* n} \subseteq \mathcal{M}$, and let $x \in \operatorname{ker} T^{* n+1}$. Then $T^{*} x \in \operatorname{ker} T^{* n} \subseteq \mathcal{M}$, and hence by the $L^{*}$-invariance $L^{*} T^{*} x \in \mathcal{M}$. Then $x=\left(I-L^{*} T^{*}\right) x+$ $L^{*} T^{*} x$ is a sum of elements from $\operatorname{ker} T^{*} \subseteq \mathcal{M}$ and $\mathcal{M}$, hence $x \in$ $\mathcal{M}$.

Corollary 2.2. Let $T \in \mathcal{B}(\mathcal{H})$ with $\|T x\| \geq\|x\|$ for all $x \in \mathcal{H}$, and let $S \subseteq \mathbb{D}$ be a set that has an accumulation point in $\mathbb{D}$. Then

$$
\bigvee_{n \geq 1} \operatorname{ker} T^{* n}=\bigvee_{\lambda \in S} \operatorname{ker}\left(T^{*}-\bar{\lambda}\right)
$$


Proof. By the previous lemma (and by taking orthocomplements) it suffices to show that

$$
\bigcap_{\lambda \in S} \operatorname{ran}(T-\lambda) \subseteq \bigcap_{\lambda \in \mathbb{D}} \operatorname{ran}(T-\lambda) .
$$

Let $x \in \bigcap_{\lambda \in S} \operatorname{ran}(T-\lambda)$, and let $L$ be the left inverse of $T$ as in the previous proof. Then $L$ is a contraction and we note that $(1-\lambda L)^{-1} x \in$ ran $T$ for every $\lambda \in S$. Let $P$ be the projection onto ran $T^{\perp}$. Then the analytic function $F(z)=P(1-z L)^{-1} x$ is zero on $S$, hence it is identically equal to zero in $\mathbb{D}$. Thus, $(1-z L)^{-1} x \in \operatorname{ran} T$ for all $z \in \mathbb{D}$, and that implies $x \in \operatorname{ran}(T-z)$ for every $z \in \mathbb{D}$.

\section{Finite RAnK EXtensions}

Lemma 3.1. Let $R \in \mathcal{B}(\mathcal{H})$ with $R^{*} R \geq I$. If $\mathcal{M} \subseteq \mathcal{H}$ is an invariant subspace of $R$ such that $U=R \mid \mathcal{M}$ is unitary, then $\mathcal{M}$ is a reducing subspace for $R$.

Proof. We have

and we calculate that

$$
R=\left[\begin{array}{ll}
U & C \\
0 & A
\end{array}\right]
$$

$$
R^{*} R-I=\left[\begin{array}{cc}
0 & U^{*} C \\
C^{*} U & C^{*} C+A^{*} A-I
\end{array}\right] \geq 0 .
$$

The positivity implies that $U^{*} C=0$, hence $C=0$.

Lemma 3.2. Let $\mathcal{H}$ be a Hilbert space, $T \in \mathcal{B}(\mathcal{H}), \lambda \in \overline{\mathbb{D}}, c_{0} \in \mathcal{H}$, and

$$
R=\left[\begin{array}{ll}
T & C \\
0 & \lambda
\end{array}\right] \text { on } \mathcal{H} \oplus \mathbb{C}
$$

where $C: \mathbb{C} \rightarrow \mathcal{H}$ satisfies $C 1=c_{0}$.

If $T$ is analytic and if $R^{*} R \geq I$, then either $R$ is analytic or $|\lambda|=1$ and there is an analytic operator $R^{\prime} \in \mathcal{B}\left(\mathcal{H}^{\prime}\right)$ such that $R$ is unitarily equivalent to $R^{\prime} \oplus \lambda$ acting on $\mathcal{H}^{\prime} \oplus \mathbb{C}$.

Proof. Suppose $R$ is not analytic and define $S=\mathbb{D} \backslash\{\lambda\}$ and $\mathcal{H}^{\prime}=$ $\bigvee_{z \in S} \operatorname{ker}\left(R^{*}-\bar{z}\right)$. Then by Corollary 2.2 we have $\mathcal{H}^{\prime \perp}=\bigcap_{z \in S} \operatorname{ran}(R-$ $z) \neq 0$. If $x \in \mathcal{H}$ such that $x \oplus 0 \in \mathcal{H}^{\prime \perp}$, then the hypothesis that $T$ is analytic implies that $x=0$. Thus, $\mathcal{H}^{\prime \perp}$ is 1 -dimensional and it contains an element of the type $x \oplus 1$, where $x \in \mathcal{H}$. We claim that $c_{0}=(\lambda-T) x$.

Let $z \in S$. Then there are $u \in \mathcal{H}$ and $\alpha \in \mathbb{C}$ such that $x=$ $(T-z) u+\alpha c_{0}$ and $1=(\lambda-z) \alpha$. Hence with some algebra we obtain

$$
c_{0}-(\lambda-T) x=(T-z)((z-\lambda) u+x) \in \operatorname{ran}(T-z)
$$


for all $z \in S$. The analyticity of $T$ implies that $c_{0}-(\lambda-T) x=0$.

Thus, $R(x \oplus 1)=\left(T x+c_{0}\right) \oplus \lambda=\lambda(x \oplus 1)$. Thus, $\lambda$ is an eigenvalue of $R$. Since $R^{*} R \geq I$ we must have $|\lambda|=1$. Then by Lemma 3.1 the eigenspace $\mathbb{C}(x \oplus 1)$ is reducing for $R$.

Set $R^{\prime}=R \mid \mathcal{H}^{\prime}$. It is clear that $\operatorname{ker}\left(R^{\prime *}-\bar{z}\right)=\operatorname{ker}\left(R^{*}-\bar{z}\right)$ for all $z \in S$. Hence by Corollary 2.2 and the definition of $\mathcal{H}^{\prime}$ it follows that $R^{\prime}$ is analytic.

In the following we will use the convention that any operator on a 0-dimensional space is both unitary and analytic. Thus, when we consider operators of the type $R=U \oplus T$, where $U$ is unitary and $T$ is analytic, then this includes the cases where either summand may be absent.

Theorem 3.3. Let $R \in \mathcal{B}(\mathcal{H})$ such that $R^{*} R \geq I$ and $\sigma(R) \subseteq \overline{\mathbb{D}}$.

If $\mathcal{M} \subseteq \mathcal{H}$ is a $R$-invariant subspace of finite codimension and such that $T=R \mid \mathcal{M}$ is analytic, then $R=U \oplus T$, where $U$ is unitary and $T$ is analytic.

Proof. $R$ has a representation as a $2 \times 2$ operator matrix of the following form

$$
R=\left[\begin{array}{ll}
T & C \\
0 & A
\end{array}\right] \text { with respect to } \mathcal{H}=\mathcal{M} \oplus \mathcal{M}^{\perp} .
$$

Set $\mathcal{M}_{0}=\mathcal{M}$ and write $R_{0}=T$. Since $A$ acts on a finite dimensional space there is an orthonormal set $\mathcal{B}=\left\{e_{1}, \ldots, e_{n}\right\}$ such that the matrix for $A$ with respect to $\mathcal{B}$ is in upper triangular form with $\lambda_{1}, \ldots, \lambda_{n}$ on the diagonal. The complex conjugates of these numbers are eigenvalues for $A^{*}$ and hence they are eigenvalues for $R^{*}$, so we must have $\left\{\lambda_{1}, \ldots, \lambda_{n}\right\} \subseteq \sigma(R) \subseteq \overline{\mathbb{D}}$.

For $1 \leq j \leq n$ set $\mathcal{M}_{j}=\mathcal{M}_{0} \oplus \operatorname{span}\left\{e_{1}, \ldots, e_{j}\right\}$ and $R_{j}=R \mid \mathcal{M}_{j}$. We will show inductively that for each $j$ we have $R_{j}=U_{j} \oplus T_{j}$, where $U_{j}$ is unitary and $T_{j}$ is analytic. Of course, $R_{j}^{*} R_{j} \geq I$ for each $0 \leq j \leq n$.

Since $R_{0}=T$ we know that $R_{0}$ is analytic, hence $R_{1}=U_{1} \oplus T_{1}$ is of the required form by Lemma 3.2. Suppose that $2 \leq j \leq n$ and that either $R_{j-1}$ is analytic or $R_{j-1}=U_{j-1} \oplus T_{j-1}$, where $U_{j-1}$ is unitary and $T_{j-1}$ is analytic. If $R_{j-1}$ is analytic, then the conclusion follows from Lemma 3.2. Suppose now that $R_{j-1}=U_{j-1} \oplus T_{j-1}$. Then the space that $U_{j-1}$ acts on is invariant for $R_{j}$, hence by Lemma 3.1 it is reducing for $R_{j}$. Hence $R_{j}$ is of the form

$$
R_{j}=\left[\begin{array}{ccc}
U_{j-1} & 0 & 0 \\
0 & T_{j-1} & C_{j} \\
0 & 0 & \lambda_{j}
\end{array}\right]
$$


Thus, we may apply Lemma $\left[3.2\right.$ to $\left[\begin{array}{cc}T_{j-1} & C_{j} \\ 0 & \lambda_{j}\end{array}\right]$ and conclude that it is either analytic or a direct sum of an analytic operator and a 1dimensional unitary. In either case, it follows that $R_{j}$ is a direct sum of a unitary and an analytic operator. This concludes the induction and it proves the theorem since $R=R_{n}$.

If $T^{*} T \geq I$, then we can form the smallest $T^{*}$-invariant subspace that contains the range of $\Delta=T^{*} T-I$. We write $\mathcal{N}_{T}=[\operatorname{ran} \Delta]_{T^{*}}$ to denote this space. Note that the orthocomplement of this space is the largest $T$-invariant subspace $\mathcal{M}$ such that $T \mid \mathcal{M}$ is isometric.

Theorem 3.4. Let $T \in \mathcal{B}(\mathcal{H})$ such that $T^{*} T \geq I$ and $\sigma(T) \subseteq \overline{\mathbb{D}}$. If $\mathcal{N}_{T}=[\operatorname{ran} \Delta]_{T^{*}}$ is finite dimensional, then $T=U \oplus T_{1}$, where $U$ is unitary and $T_{1}$ is analytic.

In fact, if $P$ denotes the projection onto $\mathcal{N}_{T}$, then

$$
T=V \oplus R,
$$

where $V$ is isometric and $R$ is analytic with $\operatorname{dim} \operatorname{ker} R^{*}=\operatorname{rank}(I-$ $P) T P<\infty$.

Let $V$ be isometric. Then by the classical Wold decomposition theorem $V=U \oplus S$, where $U$ is unitary, and $S$ is a unilateral shift (which is analytic). Then for any analytic operator $R$ the operator $T_{1}=S \oplus R$ is analytic as well. Hence the first sentence of the theorem follows from the last sentence of the theorem.

Proof. Let $\mathcal{M}=\mathcal{N}_{T}^{\perp}$. Then as remarked above $W=T \mid \mathcal{M}$ is isometric, and $T$ has a $2 \times 2$ operator matrix representation of the type

$$
T=\left[\begin{array}{rr}
W & C \\
0 & A
\end{array}\right]
$$

As in the proof of Lemma 3.1 we compute

$$
T^{*} T-I=\left[\begin{array}{cc}
0 & W^{*} C \\
C^{*} W & C^{*} C+A^{*} A-I
\end{array}\right] \geq 0,
$$

and the positivity implies that $W^{*} C=0$. In this case that implies ran $C \subseteq \operatorname{ker} W^{*}$. By the Wold decomposition theorem $W$ is a direct sum of a unitary operator $U$ and a unilateral shift. The unilateral shift part can be viewed as a direct sum of two unilateral shifts $S$ and $S^{\prime}$ where $\operatorname{ker} S^{*}=\operatorname{ran} C$ (which is finite dimensional). Thus, $W=V \oplus S$ where $V=U \oplus S^{\prime}$ is isometric. Hence $T$ has the following $3 \times 3$ operator 
matrix representation

$$
T=\left[\begin{array}{lll}
V & 0 & 0 \\
0 & S & C \\
0 & 0 & A
\end{array}\right]
$$

It now follows from Theorem 3.3 that $R=\left[\begin{array}{cc}S & C \\ 0 & A\end{array}\right]=U_{0} \oplus R_{0}$ for a unitary $U_{0}$ and an analytic operator $R_{0}$. But by our construction $R$ does not have a nontrivial unitary direct summand. Hence it must be analytic.

We have to show that $\operatorname{dim} \operatorname{ker} R^{*}=\operatorname{rank}(I-P) T P<\infty$. Note that $C=(I-P) T P$ and clearly this has finite rank, since $P$ has finite rank. Furthermore, by construction rank $C=\operatorname{dim} \operatorname{ker} S^{*}$. We will finish the proof by showing that $\operatorname{dim} \operatorname{ker} R^{*}=\operatorname{dim} \operatorname{ker} S^{*}$. Let $z \in \mathbb{D} \backslash \sigma(A)$. Then $\operatorname{dim} \operatorname{ker} R^{*}=\operatorname{dim} \operatorname{ker}\left(R^{*}-\bar{z}\right), \operatorname{dim} \operatorname{ker} S^{*}=\operatorname{dim} \operatorname{ker}\left(S^{*}-\bar{z}\right)$, and

$$
\operatorname{ker}\left(R^{*}-\bar{z}\right)=\left\{x \oplus-\left(A^{*}-\bar{z}\right)^{-1} C^{*} x: x \in \operatorname{ker}\left(S^{*}-\bar{z}\right)\right\} .
$$

The theorem follows.

\section{De Branges-Rovnyak spaces and expansive operators}

Let $\mathcal{E}$ and $\mathcal{D}$ be complex Hilbert spaces, let $H^{2}(\mathcal{D})$ be the space of square summable power series with coefficients in $\mathcal{D}$ and let $\mathcal{S}(\mathcal{D}, \mathcal{E})$ be the Schur class functions, i.e. the collection of functions that are analytic on $\mathbb{D}$ take values in the contractive operators in $\mathcal{B}(\mathcal{D}, \mathcal{E})$. A function $K: \mathbb{D} \times \mathbb{D} \rightarrow \mathcal{B}(\mathcal{E})$ is called a kernel, if it is positive definite in the sense that whenever $n \in \mathbb{N}$ and $x_{1}, \ldots, x_{n} \in \mathcal{E}, z_{1}, \ldots, z_{n} \in \mathbb{D}$, then $\sum_{i, j=1}^{n}\left\langle K\left(z_{i}, z_{j}\right) x_{i}, x_{j}\right\rangle \geq 0$. It is well-known that if $K$ is a kernel, then there is a unique Hilbert space $\mathcal{H}$ of functions on $\mathbb{D}$ with values in $\mathcal{E}$ such that for each $z \in \mathbb{D}$ and $x \in \mathcal{E}$ the function $K_{z} x=K(\cdot, z) x \in \mathcal{H}$ and $\langle f(z), x\rangle_{\mathcal{E}}=\left\langle f, K_{z} x\right\rangle_{\mathcal{H}}$ for all $f \in \mathcal{H}$.

It is also well-known that if $B \in \mathcal{S}(\mathcal{D}, \mathcal{E})$ is a Schur function, then

$$
K_{w}^{B}(z)=\frac{I_{\mathcal{E}}-B(z) B(w)^{*}}{1-z \bar{w}}
$$

is a kernel. The corresponding reproducing kernel Hilbert space is the de Branges-Rovnyak space $\mathcal{H}(B)$. The backward shift $L f(z)=$ $\frac{f(z)-f(0)}{z}$ acts contractively on each de Branges-Rovnyak space, and $\mathcal{H}(\stackrel{z}{B})$ is contractively contained in $H^{2}(\mathcal{E})$. Note that if $B(0)=0$, then $K_{0}^{B}(z)=I_{\mathcal{E}}$, hence the constant functions are contained in $\mathcal{H}(B)$, $\mathcal{E} \subseteq \mathcal{H}(B)$, and in fact ker $L=\mathcal{E}$. We will need a few further facts about the connections between $L, B$, and $K_{w}^{B}(z)$ in the case when $B(0)=0$. 
Lemma 4.1. Let $L_{0} \in \mathcal{B}(\mathcal{H})$ be a Hilbert space contraction, let $D_{*}=$ $\left(I-L_{0} L_{0}^{*}\right)^{1 / 2}, \mathcal{D}_{*}=\overline{\operatorname{ran} D_{*}}$, and let $P$ denote the projection onto $\mathcal{E}_{*}=\operatorname{ker} L_{0}$.

Then

$$
B(z)=z P\left(1-z L_{0}\right)^{-1} D_{*} \mid \mathcal{D}_{*}
$$

is in the Schur class $\mathcal{S}\left(\mathcal{D}_{*}, \mathcal{E}_{*}\right)$. B satisfies $B(0)=0$ and

$$
K_{w}^{B}(z)=P\left(1-z L_{0}\right)^{-1}\left(1-\bar{w} L_{0}^{*}\right)^{-1} P .
$$

Furthermore, the map $V$,

$$
V x(z)=P\left(I-z L_{0}\right)^{-1} x
$$

defines a partial isometry of $\mathcal{H}$ onto $\mathcal{H}(B)$. It satisfies $V L_{0}=L V$ and $V D_{*} x(z)=\frac{B(z)}{z} x$ for all $x \in \mathcal{H}$ and $z \in \mathbb{D}$.

Proof. It is clear that $B(0)=0$ and the fact that $B$ is in the Schur class will follow once we establish the identity $K_{w}^{B}(z)=P\left(1-z L_{0}\right)^{-1}(1-$ $\left.\bar{w} L_{0}^{*}\right)^{-1} P$ since the expression on the right is clearly a kernel. However, for the bigger picture it is useful to note that the colligation operator $U: \mathcal{E}_{*} \oplus \mathcal{H} \rightarrow \mathcal{D}_{*} \oplus \mathcal{H}$ defined by

$$
U=\left[\begin{array}{ll}
0 & D_{*} \\
P & L_{0}^{*}
\end{array}\right]
$$

is isometric, and hence

$$
B(z)^{*}=\bar{z} D_{*}\left(I-\bar{z} L_{0}^{*}\right)^{-1} P
$$

is the transfer function realization of the Schur function $B \in \mathcal{S}\left(\mathcal{D}_{*}, \mathcal{E}_{*}\right)$, see e.g. 2], Corollary 8.26.

Write $K_{w}(z)=P\left(1-z L_{0}\right)^{-1}\left(1-\bar{w} L_{0}^{*}\right)^{-1} P$, and for later reference note that $P L_{0}^{*}=L_{0} P=0$ implies that

$$
K_{w}(z)=P+P\left(I-z L_{0}\right)^{-1}\left(z L_{0}\right)\left(\bar{w} L_{0}^{*}\right)\left(I-\bar{w} L_{0}^{*}\right)^{-1} P .
$$

Now calculate

$$
\begin{aligned}
B(z) B(w)^{*} & =z \bar{w} P\left(I-z L_{0}\right)^{-1} D_{*}^{2}\left(I-\bar{w} L_{0}^{*}\right)^{-1} P \\
& =z \bar{w} P\left(I-z L_{0}\right)^{-1}\left(I-L_{0} L_{0}^{*}\right)\left(I-\bar{w} L_{0}^{*}\right)^{-1} P \\
& =z \bar{w} K_{w}(z)-\left(P\left(I-z L_{0}\right)^{-1}\left(z L_{0}\right)\left(\bar{w} L_{0}^{*}\right)\left(I-\bar{w} L_{0}^{*}\right)^{-1} P\right) \\
& =z \bar{w} K_{w}(z)-\left(K_{w}(z)-P\right) \text { by (4.1) } \\
& =P-(1-z \bar{w}) K_{w}(z)
\end{aligned}
$$

Noting that $P$ acts as the identity on $\mathcal{E}_{*}$ we obtain $K_{w}(z)=\frac{I-B(z) B(w)^{*}}{1-z \bar{w}}=$ $K_{w}^{B}(z)$.

The linear function $V$ defined on $\mathcal{H}$ by $V x(z)=P\left(I-z L_{0}\right)^{-1} x$ takes values in $\operatorname{Hol}(\mathbb{D}, \mathcal{E})$, the analytic $\mathcal{E}$-valued functions on $\mathbb{D}$. The kernel 
of $V$ equals $\bigcap_{n \geq 0}$ ker $P L_{0}^{n}$. In order to show that $V$ is a partial isometry into $\mathcal{H}(B)$, it suffices to show that $V$ is isometric on the set

$$
\left(\bigcap_{n \geq 0} \operatorname{ker} P L_{0}^{n}\right)^{\perp}=\operatorname{Span}_{n \geq 0} \operatorname{ran}\left(L_{0}^{*}\right)^{n} P=\operatorname{Span}_{|w|<1} \operatorname{ran}\left(I-\bar{w} L_{0}^{*}\right)^{-1} P \text {. }
$$

But if $x=\sum_{i=1}^{n}\left(I-\overline{w_{i}} L_{0}^{*}\right)^{-1} P x_{i}$ for some $w_{1}, \ldots, w_{n} \in \mathbb{D}$ and $x_{1}, \ldots x_{n} \in$ $\mathcal{H}$, then

$$
V x(z)=\sum_{i=1}^{n} K_{w_{i}}^{B}(z) x_{i} \in \mathcal{H}(B)
$$

and

$$
\begin{aligned}
\|V x\|_{\mathcal{H}(B)}^{2} & =\sum_{i, j=1}^{n}\left\langle K_{w_{i}}^{B}\left(w_{j}\right) x_{i}, x_{j}\right\rangle \\
& =\sum_{i, j=1}^{n}\left\langle P\left(1-w_{i} L_{0}\right)^{-1}\left(I-\bar{w}_{j} L_{0}^{*}\right)^{-1} P x_{i}, x_{j}\right\rangle \\
& =\left\|\sum_{i=1}^{n}\left(I-\bar{w}_{i} L_{0}^{*}\right)^{-1} P x_{i}\right\|^{2} \\
& =\|x\|^{2} .
\end{aligned}
$$

Thus, $V$ defines a partial isometry and it must be onto by (4.2). The identities $V L_{0}=L V$ and $V D_{*} x(z)=\frac{B(z)}{z} x$ for all $x \in \mathcal{H}$ and $z \in \mathbb{D}$ follow easily.

Remark 4.2. A main result of de Branges and Rovnyak states that every Hilbert space contraction $X$ that is completely non-isometric, i.e.

$$
\left\{x \in \mathcal{H}:\left\|X^{n} x\right\|=\|x\| \text { for all } n \geq 0\right\}=\{0\},
$$

is unitarily equivalent to a backward shift acting on some $\mathcal{H}(B)$ space (and conversely).

That result can be seen to follow from our construction as follows. Given a completely non-isometric contraction $X$, let $D=\left(I-X^{*} X\right)^{1 / 2}$, $\mathcal{E}=\overline{\operatorname{ran} D}$, then the operator

$$
L_{0}=\left[\begin{array}{ll}
X & 0 \\
D & 0
\end{array}\right] \text { acting on } \mathcal{H} \oplus \mathcal{E}
$$

is a contraction, and if we apply the construction of the previous lemma to $L_{0}$, then the resulting $V: \mathcal{H} \oplus \mathcal{E} \rightarrow \mathcal{H}(B)$ is 1 -1, hence unitary. Furthermore, one easily checks that $X$ is unitarily equivalent to $\left(L, \mathcal{H}\left(B_{1}\right)\right)$, where $B_{1}(z)=B(z) / z$. We omit the details. 
For the forward shift $Y: f \rightarrow z f$ to act boundedly on $\mathcal{H}(B)$ an extra condition is required. For scalar Schur functions $b$ the condition is that $b$ is not an extreme point of the unit ball of $H^{\infty}$, or equivalently $\log (1-|b|) \in L^{1}(\mathbb{T})$. The condition turns out to be equivalent to the condition that $b \in \mathcal{H}(b)$. For operator-valued Schur functions the following lemma describes the analogue of this condition for the case where $B(0)=0$.

Lemma 4.3. Let $B \in \mathcal{S}\left(\mathcal{D}_{*}, \mathcal{E}\right)$ with $B(0)=0$, then the following are equivalent:

(i) $Y: f \rightarrow z f$ defines a bounded operator on $\mathcal{H}(B)$,

(ii) for each $x \in \mathcal{D}_{*}$ the function $g_{x}(z)=B(z) x \in \mathcal{H}(B)$.

Indeed, if $x \rightarrow \tau(x)$ is defined by $\tau(x)(z)=B(z) x$, then $Y$ is bounded on $\mathcal{H}(B)$, if and only if $\tau: \mathcal{D}_{*} \rightarrow \mathcal{H}(B)$ is bounded with $\|\tau\|^{2}=$ $\|Y\|^{2}-1$.

Proof. Let $0<c<1$, then one verifies the identity

$$
\begin{aligned}
\frac{I-B(z) B(w)^{*}}{1-z \bar{w}} & -\frac{c}{1-c} B(z) B(w)^{*}-I \\
& =\frac{1}{1-c}\left(\frac{(1-c z \bar{w})\left(I-B(z) B(w)^{*}\right)}{1-z \bar{w}}-I\right) .
\end{aligned}
$$

Note that if $K_{w}^{B}(0)=I$, then $K_{w}^{B}(z)$ is positive definite, if and only if $K_{w}^{B}(z)-I$ is positive definite. Hence it follows that

$$
\frac{(1-c z \bar{w})\left(I-B(z) B(w)^{*}\right)}{1-z \bar{w}}
$$

is positive definite, if and only if

$$
\frac{I-B(z) B(w)^{*}}{1-z \bar{w}}-\frac{c}{1-c} B(z) B(w)^{*}
$$

is positive definite. The first condition (4.3) is equivalent to $Y$ being bounded with $\|Y\|^{2} \leq 1 / c$, while the second condition (4.4) is equivalent to the map $x \rightarrow \tau(x), \tau(x)(z)=B(z) x$ being bounded with $\|\tau\|^{2} \leq \frac{1}{c}-1$. Indeed, for (4.4) one checks that the positivity of $\frac{I-B(z) B(w)^{*}}{1-z \bar{w}}-\frac{c}{1-c} B(z) B(w)^{*}$ is equivalent to the inequality

$$
\left\|\sum_{i} B\left(z_{i}\right)^{*} x_{i}\right\|^{2} \leq\left(\frac{1}{c}-1\right)\left\|\sum_{i} K_{z_{i}}^{B}(\cdot) x_{i}\right\|^{2},
$$

which gives the norm inequality for $\tau^{*}$. 
Example 4.4. The Dirichlet space $D$ has reproducing kernel

$$
k_{w}(z)=\frac{1}{\bar{w} z} \log \frac{1}{1-\bar{w} z}=\sum_{n=0}^{\infty} \frac{\bar{w}^{n} z^{n}}{n+1} .
$$

In this case one can take $B(z)=\left\{b_{n}\right\}_{n \geq 1}, b_{n}(z)=\frac{z^{n}}{\sqrt{n(n+1)}}$. Then for $x=\left\{a_{n}\right\} \in \ell^{2}$ we have $B(z) x=\sum_{n \geq 1} a_{n} b_{n}(z)=\sum_{n \geq 1} \frac{a_{n} z^{n}}{\sqrt{n(n+1)}} \in D$. Note that $\left\|B\left(e^{i t}\right)\right\|=1$ for each $t$. Thus, in this case $M_{z}$ is bounded although $\log \left(1-\left\|B\left(e^{i t}\right)\right\|\right) \notin L^{1}(\mathbb{T})$.

Let $B$ be any Schur function. Then since $X$ is a contraction, if $M_{z}$ acts boundedly on $\mathcal{H}(B)$, then $\left\|M_{z} f\right\| \geq\left\|X M_{z} f\right\|=\|f\|$ for all $f \in \mathcal{H}(B)$, i.e. $M_{z}$ is norm expansive. Let $T \in \mathcal{B}(\mathcal{H})$ be such that $\|T x\| \geq\|x\|$ for all $x \in \mathcal{H}$. Then $\operatorname{ran} T^{n}$ is closed for each $n$, and recall that $T$ is analytic, if $\bigcap_{n>0} \operatorname{ran} T^{n}=(0)$. Thus, whenever $M_{z}$ is bounded on $\mathcal{H}(B)$, then as $\mathcal{H}(B) \subseteq H^{2}(\mathcal{E})$ it is obvious that $M_{z}$ is analytic. The next theorem says that all norm expansive analytic operators can be modelled as $M_{z}$ on some $\mathcal{H}(B)$.

Lemma 4.5. If $T \in \mathcal{B}(\mathcal{H})$ satisfies $\Delta=T^{*} T-I \geq 0$, and if $L=$ $\left(T^{*} T\right)^{-1} T^{*}$ is the left inverse of $T$ with $\operatorname{ker} L=\operatorname{ker} T^{*}$, then

$$
\overline{\operatorname{ran} \Delta}=\overline{\operatorname{ran} D_{*}}, \quad D_{*}=\left(I-L L^{*}\right)^{1 / 2} .
$$

Proof. The lemma follows from the identity $D_{*}^{2}=I-\left(T^{*} T\right)^{-1}=I-$ $(I+\Delta)^{-1}=\Delta(I+\Delta)^{-1}$.

Theorem 4.6. Let $T \in \mathcal{B}(\mathcal{H})$. Then the following are equivalent

(i) $T$ is analytic and norm expanding,

(ii) there are Hilbert spaces $\mathcal{E}$ and $\mathcal{D}$, a Schur function $B \in \mathcal{S}(\mathcal{D}, \mathcal{E})$ such that $B(0)=0, M_{z} \in \mathcal{B}(\mathcal{H}(B))$, and $T$ is unitarily equivalent to $\left(M_{z}, \mathcal{H}(B)\right)$,

(iii) there are Hilbert spaces $\mathcal{E}$ and $\mathcal{D}$, a Schur function $B \in \mathcal{S}(\mathcal{D}, \mathcal{E})$, $M_{z} \in \mathcal{B}(\mathcal{H}(B))$, and $T$ is unitarily equivalent to $\left(M_{z}, \mathcal{H}(B)\right)$.

If the conditions are satisfied, then in (ii) one can take $\mathcal{E}=\operatorname{ker} T^{*}$ and $\mathcal{D}=\overline{\operatorname{ran}\left(T^{*} T-I\right)}$.

Proof. (ii) $\Rightarrow$ (iii) is trivial and we already noted that (iii) $\Rightarrow$ (i).

(i) $\Rightarrow$ (ii): Let $T$ be analytic and norm expanding. Then $T^{*} T$ is invertible and $L=\left(T^{*} T\right)^{-1} T^{*}$ is a left inverse of $T$ with $\mathcal{E}=\operatorname{ker} L=$ $\operatorname{ker} T^{*}$. The operator $L^{*}$ has been called the Cauchy dual of $T$, see [37]. Note that $I-T L=I-L^{*} T^{*}=P$, the projection onto $\mathcal{E}$. Since $\mathcal{H}=\operatorname{ran} T \oplus \operatorname{ker} T^{*}$ one easily sees that $\|L\| \leq 1$. Thus, as in Lemma 4.1 for any $x \in \mathcal{H}$ one can define the $\mathcal{E}$-valued holomorphic function 
$V x: \mathbb{D} \rightarrow \mathcal{E}$ by $V x(z)=P(I-z L)^{-1} x, z \in \mathbb{D}$. In [37], Lemma 2.2, Shimorin showed that the analyticity of $T$ implies that $V$ is $1-1$, and hence by Lemma $4.1 V: \mathcal{H} \rightarrow \mathcal{H}(B)$ is unitary and one easily verifies that $V T=M_{z} V$.

By Lemma 4.1 we can take $B \in \mathcal{S}\left(\overline{\operatorname{ran} D_{*}}\right.$, ker $\left.L\right)$. We already noted that $\operatorname{ker} T^{*}=\operatorname{ker} L$ and by Lemma 4.5 we have $\overline{\operatorname{ran}\left(1-L L^{*}\right)^{1 / 2}}=$ $\overline{\operatorname{ran}\left(T^{*} T-I\right)}$.

Theorem4.6implies that for a study of operators of the type $\left(M_{z}, \mathcal{H}(B)\right)$ there is no loss in generality, if we assume that $B(0)=0$. In the remainder of this paper that will be the standard assumption. Of course, that means that all hypotheses and conclusions about $B$ and the mate $a$ are only valid if $B(0)=0$. Thus, it will be useful to know how to pass to the general case. We will now explain how to do this for scalarvalued Schur functions $B$. In fact, it turns out that for two contractive analytic functions $B=\left(b_{1}, b_{2}, \ldots\right)$ and $C=\left(c_{1}, c_{2}, \ldots\right)$ the operators $\left(M_{z}, \mathcal{H}(B)\right)$ and $\left(M_{z}, \mathcal{H}(C)\right)$ are unitarily equivalent to one another, if and only if $B$ and $C$ are related by a ball automorphism.

For $k \in \mathbb{N} \cup\{\infty\}$ let $\mathbb{B}_{k}$ be the unit ball in $\mathbb{C}^{k}$ (or $\ell_{2}$ if $k=\infty$ ), and for $\alpha \in \mathbb{B}_{k}$ let $\varphi_{\alpha}$ be the analytic automorphism $\mathbb{B}_{k} \rightarrow \mathbb{B}_{k}$ defined by $\varphi_{\alpha}(z)=-z$, if $\alpha=0$ and

$$
\varphi_{\alpha}(z)=\frac{\alpha-P_{\alpha} z-\left(1-\|\alpha\|^{2}\right)^{1 / 2} Q_{\alpha} z}{1-\langle z, \alpha\rangle},
$$

if $\alpha \in \mathbb{B}_{k} \backslash\{0\}$. Here $P_{\alpha} z=\frac{\langle z, \alpha\rangle}{|\alpha|^{2}} \alpha, Q_{\alpha}=I_{\mathbb{C}^{k}}-P_{\alpha}$. It satisfies $\varphi_{\alpha}(\alpha)=0, \varphi_{\alpha}(0)=\alpha, \varphi_{\alpha}^{-1}=\varphi_{\alpha}$, and

$$
1-\left\langle\varphi_{\alpha}(z), \varphi_{\alpha}(w)\right\rangle=\frac{\left(1-\|\alpha\|^{2}\right)(1-\langle z, w\rangle)}{(1-\langle z, \alpha\rangle)(1-\langle\alpha, w\rangle)}
$$

see 36.

Thus, if $B=\left(b_{1}, \ldots, b_{k}\right)$ or $B=\left(b_{1}, b_{2}, \ldots\right)$ is a $\mathbb{B}_{k}$-valued analytic function on $\mathbb{D}$, then

$$
\frac{1-\left\langle\varphi_{\alpha}(B(z)), \varphi_{\alpha}(B(w))\right\rangle}{1-z \bar{w}}=\frac{\left(1-\|\alpha\|^{2}\right)}{(1-\langle z, \alpha\rangle)(1-\langle\alpha, w\rangle)} \frac{1-\langle B(z), B(w)\rangle}{1-z \bar{w}} .
$$

This implies that if we write $B_{\alpha}(z)=\varphi_{\alpha}(B(z))$ and $f_{\alpha}(z)=\frac{\sqrt{1-\|\alpha\|^{2}}}{1-\langle z, \alpha\rangle}$, then

$$
K_{w}^{B_{\alpha}}(z)=f_{\alpha}(z) \overline{f_{\alpha}(w)} K_{w}^{B}(z) .
$$

Thus, the following lemma is obvious.

Lemma 4.7. For each $\alpha \in \mathbb{B}_{k}$ and each $\mathbb{B}_{k}$-valued analytic function $B$ the operator $M_{f_{\alpha}}: \mathcal{H}\left(K^{B}\right) \rightarrow \mathcal{H}\left(K^{B_{\alpha}}\right), g \rightarrow f_{\alpha} g$ is unitary. 
Furthermore, $T=\left(M_{z}, \mathcal{H}(B)\right)$ is bounded, if and only if $T_{\alpha}=$ $\left(M_{z}, \mathcal{H}\left(B_{\alpha}\right)\right)$ is bounded and $T_{\alpha} M_{f_{\alpha}}=M_{f_{\alpha}} T$.

We conclude that if a $\mathbb{B}_{k}$-valued analytic function $B$ is given, and if $T=\left(M_{z}, \mathcal{H}(B)\right)$ is a bounded operator, then by taking $\alpha=B(0)$ we obtain a $B_{\alpha}$ with $B_{\alpha}(0)=0$ and $T$ is unitarily equivalent to $\left(M_{z}, \mathcal{H}\left(B_{\alpha}\right)\right)$.

The next lemma can be considered to be a converse of the above observation.

Lemma 4.8. Let $k, j \in \mathbb{N} \cup\{\infty\}$, let $B$ be $\mathbb{B}_{k}$-valued analytic function with $B(0)=0$, and let $C$ be a $\mathbb{B}_{j}$-valued analytic function with $C(0)=$ 0 .

If $T=\left(M_{z}, \mathcal{H}(B)\right)$ and $S=\left(M_{z}, \mathcal{H}(C)\right)$ are bounded and unitarily equivalent to one another, then $K_{w}^{C}(z)=K_{w}^{B}(z)$ for all $z, w \in \mathbb{D}$ and there is a partial isometry $V: \mathbb{C}^{k} \rightarrow \mathbb{C}^{j}$ (or $\ell_{2} \rightarrow \ell_{2}$, etc.) such that $C(z)=V(B(z))$ for each $z \in \mathbb{D}$.

Proof. Suppose $T=\left(M_{z}, \mathcal{H}(B)\right)$ is a bounded operator. We claim that for each $\lambda \in \mathbb{D}$ we have $\operatorname{ker}(T-\lambda)^{*}=\left\{\gamma K_{\lambda}^{B}: \gamma \in \mathbb{C}\right\}$. It is clear that $K_{\lambda}^{B} \in \operatorname{ker}(T-\lambda)^{*}$, thus it suffices to show that $\operatorname{dim} \operatorname{ker}(T-\lambda)^{*}=1$ for each $\lambda \in \mathbb{D}$.

If $f(0)=0$, then $\frac{f(z)}{z}=\frac{f(z)-f(0)}{z}=L f(z) \in \mathcal{H}(B)$ and hence $f \in$ ran $T$. Thus, $\operatorname{dim} \operatorname{ker} T^{*}=1$ and as $T$ is expansive, the Fredholm theory implies that $\operatorname{dim} \operatorname{ker}(T-\lambda)^{*}=\operatorname{dim} \operatorname{ker} T^{*}=1$ for each $\lambda \in \mathbb{D}$. Similarly, we conclude that $\operatorname{ker}(S-\lambda)^{*}=\left\{\gamma K_{\lambda}^{C}: \gamma \in \mathbb{C}\right\}$.

Let $U: \mathcal{H}(B) \rightarrow \mathcal{H}(C)$ be unitary such that $U T=S U$. Then $U^{*}\left(\operatorname{ker}(S-\lambda)^{*}\right)=\operatorname{ker}(T-\lambda)^{*}$ for each $\lambda \in \mathbb{D}$. This implies that $U^{*} K_{\lambda}^{C}=\overline{f(\lambda)} K_{\lambda}^{B}$ for some value $f(\lambda) \in \mathbb{C}$. This leads to the equality

$$
\frac{1-\langle C(z), C(w)\rangle}{1-z \bar{w}}=f(z) \overline{f(w)} \frac{1-\langle B(z), B(w)\rangle}{1-z \bar{w}}
$$

for all $z, w \in \mathbb{D}$. Taking $z=w=0$ we see that the hypothesis that $C(0)=B(0)=0$ implies that $f(0)$ has modulus 1 . Similarly, we take $w=0$, and conclude that for all $z \in \mathbb{D}$ the identity $1=f(z) \overline{f(0)}$ holds, so $f$ must be constant. This implies that $\langle C(z), C(w)\rangle=\langle B(z), B(w)\rangle$ for all $z, w \in \mathbb{D}$. Thus $K^{C}=K^{B}$.

Let $\mathcal{M}=\bigvee\{B(z): z \in \mathbb{D}\} \subseteq \mathbb{C}^{k}$ (or $\ell_{2}$ ), and define a linear transformation $V: \mathcal{M} \rightarrow \mathbb{C}^{j}\left(\operatorname{resp} . V: \mathcal{M} \rightarrow \ell_{2}\right)$ by $V(B(z))=C(z)$. The identity $\langle C(z), C(w)\rangle=\langle B(z), B(w)\rangle$ implies that $V$ is well-defined and isometric. It becomes a partial isometry on $\mathbb{C}^{k}$, if we set it equal to 0 on $\mathcal{M}^{\perp}$. This concludes the proof of the lemma. 
5. Some observations about Schur functions with $B(0)=0$.

Note that if $B \in \mathcal{S}(\mathcal{D}, \mathcal{E})$ is such that $B(0)=0$, then $K_{0}^{B}(z)=I_{\mathcal{E}}$ and this implies that the constant functions form the orthocomplement in $\mathcal{H}(B)$ of the functions that are 0 at 0 . Obviously the constant functions form the null space of $L$, the backward shift on $\mathcal{H}(B)$. If $T=\left(M_{z}, \mathcal{H}(B)\right)$ is bounded, then this implies that $\operatorname{ker} T^{*}=\operatorname{ker} L$. Since the operator $\left(T^{*} T\right)^{-1} T^{*}$ is a left inverse of $T$ with null space equal to $\operatorname{ker} T^{*}$, we conclude that the hypothesis $B(0)=0$ implies that $L=\left(T^{*} T\right)^{-1} T^{*}$, i.e. the backward shift equals the operator from the construction of the proof of Theorem 4.6.

Lemma 5.1. If $B \in \mathcal{S}(\mathcal{D}, \mathcal{E})$ with $B(0)=0$ is such that $T=\left(M_{z}, \mathcal{H}(B)\right)$ defines a bounded operator on $\mathcal{H}(B)$, then

$$
\overline{\operatorname{ran} \Delta}=\operatorname{clos}\left\{g_{x}: x \in \mathcal{D}, g_{x}(z)=\frac{B(z)}{z} x\right\}, \Delta=T^{*} T-I .
$$

Proof. As above let $L$ be the backward shift on $\mathcal{H}(B)$, and $D_{*}=(I-$ $\left.L L^{*}\right)^{1 / 2}$. Then by Lemma 4.5 we have $\overline{\operatorname{ran} \Delta}=\overline{\operatorname{ran} D_{*}}$.

If $y \in \mathcal{E}$ and $w \in \mathbb{D}$, then set $f=K_{w}^{B}(\cdot) y$. One calculates that $\left(\left(I-L L^{*}\right) f\right)(z)=\frac{B(z)}{z} \frac{B(w)^{*}}{\bar{w}} y$. Since elements of the type as $f$ span $\mathcal{H}(B)$ we have

$$
\begin{aligned}
\overline{\operatorname{ran} D_{*}} & =\overline{\operatorname{ran} D_{*}^{2}} \\
& =\bigvee_{w \in \mathbb{D}}\left\{g_{w, y}: g_{w, y}(z)=\frac{B(z)}{z} \frac{B(w)^{*}}{\bar{w}} y, y \in \mathcal{E}\right\} \\
& =\operatorname{clos}\left\{g_{x}: x \in \mathcal{D}, g_{x}(z)=\frac{B(z)}{z} x\right\},
\end{aligned}
$$

where the last equality follows, because the function $\frac{B(z)}{z} a$ is identically equal to 0 , if $a \perp \operatorname{ran} \frac{B(w)^{*}}{\bar{w}}$ for all $w \in \mathbb{D}$.

Lemma 5.2. Let $B \in \mathcal{S}(\mathcal{D}, \mathcal{E})$ be such that $B(0)=0$ and such that $T=\left(M_{z}, \mathcal{H}(B)\right)$ is bounded.

If $\mathcal{M}$ is a reducing subspace for $T$, and if $\mathcal{E}_{0}=\mathcal{M} \cap \operatorname{ker} T^{*}$, then $f(z) \in \mathcal{E}_{0}$ for every $f \in \mathcal{M}$ and $z \in \mathbb{D}$, and $\mathcal{E}_{0}$ is reducing for $K_{w}^{B}(z)$ and for $B(z) B(w)^{*}$ for every $z, w \in \mathbb{D}$.

Proof. As before the backward shift $L$ satisfies $L=\left(T^{*} T\right)^{-1} T^{*}$ and $L^{*}=T\left(T^{*} T\right)^{-1}$. Thus the hypothesis that $\mathcal{M}$ is reducing for $T$ implies that it also reduces $L$. Hence, if $f \in \mathcal{M}$, then $f(0)=f-T L f \in$ $\mathcal{M} \cap \operatorname{ker} T^{*}=\mathcal{E}_{0}$. If for such $f$ we have $f(z)=\sum_{n=0}^{\infty} a_{n} z^{n}$, then for each $n, a_{n}=L^{n} f(0) \in \mathcal{E}_{0}$. This implies $f(z) \in \mathcal{E}_{0}$ for all $z \in \mathbb{D}$. 
By symmetry we have $f(w) \in \mathcal{E} \ominus \mathcal{E}_{0}$ for all $f \in \mathcal{M}^{\perp}$ and $w \in \mathbb{D}$. Let $x \in \mathcal{E}_{0}$, then for every $f \in \mathcal{M}^{\perp}$ and $w \in \mathbb{D}$ we have

$$
0=\langle f(w), x\rangle_{\mathcal{E}}=\left\langle f, K_{w}^{B}(\cdot) x\right\rangle_{\mathcal{H}(B)} .
$$

This implies that $K_{w}^{B}(\cdot) x \in \mathcal{M}$ and hence by the first part of the proof $K_{w}^{B}(z) x \in \mathcal{E}_{0}$ for all $z, w \in \mathbb{D}$. Thus, $\mathcal{E}_{0}$ is invariant for $K_{w}^{B}(z)$, and by symmetry it must be reducing. Next note that $B(z) B(w)^{*}=I-(1-$ $z \bar{w}) K_{w}^{B}(z)$. This implies that $\mathcal{E}_{0}$ is also reducing for $B(z) B(w)^{*}$.

Lemma 5.3. Let $B \in \mathcal{S}(\mathcal{D}, \mathcal{E})$ be such that $B(0)=0$ and such that $T=\left(M_{z}, \mathcal{H}(B)\right)$ is bounded.

Then $\mathcal{E}=\bigvee_{z \in \mathbb{D}} \operatorname{ran} B(z)$, if and only if $\left(M_{z}, \mathcal{H}(B)\right)$ has no nontrivial reducing subspace on which it acts isometrically.

Proof. Let $\mathcal{R}_{B}=\bigvee_{z \in \mathbb{D}}$ ran $B(z)$ and suppose that $\mathcal{R}_{B} \neq \mathcal{E}$. Then

$$
K_{w}^{B}(z)=\frac{1}{1-z \bar{w}} I_{\mathcal{R}_{B}^{\perp}} \oplus K_{w}^{B_{1}}(z),
$$

where $B_{1} \in \mathcal{S}\left(\mathcal{D}, \mathcal{R}_{B}\right)$ is defined by $B_{1}(z)=P_{\mathcal{R}_{B}} B(z)$. It is then clear that $\mathcal{H}(B)=H^{2}\left(\mathcal{R}_{B}^{\perp}\right) \oplus \mathcal{H}\left(B_{1}\right)$ and $\left(M_{z}, \mathcal{H}(B)\right)$ has a unilateral shift of multiplicity $\operatorname{dim} \mathcal{R}_{B}^{\perp}$ as a direct summand.

Now suppose $T$ has a nontrivial reducing subspace on which it acts isometrically. Then by Lemma 5.2 there is a nontrivial subspace $\mathcal{E}_{0}$ of $\mathcal{E}$ that reduces $B(z) B(w)^{*}$ for all $z, w \in \mathbb{D}$. Furthermore, since $T_{0}=\left(M_{z}, \mathcal{H}\left(B_{0}\right)\right)$ is isometric, if and only if $B_{0}=0$, we must have $B(z) B(w)^{*} \mid \mathcal{E}_{0}=0$ for all $z, w \in \mathbb{D}$. But then $\operatorname{ran} B(z) B(w)^{*} \subseteq \mathcal{E} \ominus \mathcal{E}_{0}$ and this implies $\mathcal{E}_{0} \perp \operatorname{ran} B(z)$ for all $z \in \mathbb{D}$.

\section{Rational matrix-VAlued Schur Class Functions}

Lemma 6.1. If $T \in \mathcal{B}(\mathcal{H})$ satisfies $\Delta=T^{*} T-I \geq 0$, and if $L=$ $\left(T^{*} T\right)^{-1} T^{*}$ is the left inverse of $T$ with $\operatorname{ker} L=\operatorname{ker} T^{*}$, then

$$
[\operatorname{ran} \Delta]_{T^{*}}=[\operatorname{ran} \Delta]_{L}
$$

Proof. We start by showing that $[\operatorname{ran} \Delta]_{T^{*}}$ is $L$-invariant. That will imply one of the inclusions and the other one will follow analogously. Let $x \in[\operatorname{ran} \Delta]_{T^{*}}$, then set $y=(I+\Delta)^{-1} T^{*} x$. Since $L=(I+\Delta)^{-1} T^{*}=$ $T^{*}-\Delta(I+\Delta)^{-1} T^{*}$ we have $L x=T^{*} x+\Delta y \in[\operatorname{ran} \Delta]_{T^{*}}$.

Similarly, if $x \in[\operatorname{ran} \Delta]_{L}$, then $T^{*} x=(I+\Delta) L x=L x+\Delta L x \in$ $[\operatorname{ran} \Delta]_{L}$.

If $\mathcal{D}$ and $\mathcal{E}$ are arbitrary Hilbert spaces and $B \in \mathcal{S}(\mathcal{D}, \mathcal{E})$, then we will say that $B$ is rational, if there is a scalar polynomial $q \neq 0$ and an operator polynomial $P$ such that $B(z)=\frac{1}{q(z)} P(z)$. Recall that the 
degree of $B$ is defined to be the smallest integer $n$ such that there are such polynomials both of which have degree $\leq n$.

Theorem 6.2. Let $B \in \mathcal{S}(\mathcal{D}, \mathcal{E})$ with $B(0)=0$ be such that $T=$ $\left(M_{z}, \mathcal{H}(B)\right)$ defines a bounded operator on $\mathcal{H}(B)$. Write $\Delta=T^{*} T-I$, $\mathcal{N}=[\operatorname{ran} \Delta]_{T^{*}}$ and $\mathcal{R}_{B}=\bigvee_{z \in \mathbb{D}} \operatorname{ran} B(z)$.

Then $\operatorname{dim} \mathcal{N}<\infty$, if and only if $B$ is rational and $\operatorname{dim} \mathcal{R}_{B}<\infty$. In fact,

$$
\text { degree } B \leq \operatorname{dim} \mathcal{N} \leq \operatorname{dim} \mathcal{R}_{B} \text { degree } B .
$$

Furthermore, if $\operatorname{dim} \mathcal{N}=n$, then there is an operator-valued polynomial $P(z)=\sum_{k=1}^{n} P_{k} z^{k}, P_{k} \in \mathcal{B}(\mathcal{D}, \mathcal{E})$ such that $B(z)=\frac{1}{\tilde{q}(z)} P(z)$, where $q$ is the characteristic polynomial of $L \mid \mathcal{N}$, and $\tilde{q}(z)=z^{n} q(1 / z)$.

Proof. If $\mathcal{R}_{B} \neq \mathcal{E}$, then by Lemmas 5.2 and $5.3 T$ will have a nontrivial reducing subspace $\mathcal{M}$ on which it acts isometrically. This subspace will be reducing for $L$ and $\Delta$, and $\Delta \mid \mathcal{M}=0$ and $\mathcal{N} \subseteq \mathcal{M}^{\perp}$. Thus, there will be no loss in generality, if we assume that $\mathcal{E}=\mathcal{R}_{B}$.

Suppose $\mathcal{N}=[\operatorname{ran} \Delta]_{T^{*}}$ has dimension $n$. Then by Lemma 6.1 $\mathcal{N}$ is invariant for $L$, hence $L \mid \mathcal{N}$ can be represented by an $n \times n$ matrix. Let $q(z)=\sum_{j=0}^{n} \hat{q}_{j} z^{j}$ be the characteristic polynomial of this matrix. Let $x \in \mathcal{D}$, then by Lemma 5.1 the function $g(z)=\frac{B(z)}{z} x \in \overline{\operatorname{ran} \Delta}$ and hence $q(L) g=0$. Let $B(z)=\sum_{n=1}^{\infty} B_{n} z^{n}$ for $B_{n} \in \mathcal{B}(\mathcal{D}, \mathcal{E})$. Then for each $j \in \mathbb{N}$ we have $z^{j}\left(L^{j} g\right)(z)=\frac{\bar{B}(z)}{z} x-\sum_{k=1}^{j}\left(B_{k} x\right) z^{k-1}$. Hence

$$
\begin{aligned}
0=z^{n}(q(L) g)(z) & =\sum_{j=0}^{n} \hat{q}_{j} z^{n-j} z^{j}\left(L^{j} g\right)(z) \\
& =z^{n} q(1 / z) \frac{B(z)}{z} x-\sum_{j=0}^{n} \hat{q}_{j} z^{n-j} \sum_{k=1}^{j}\left(B_{k} x\right) z^{k-1} .
\end{aligned}
$$

Thus,

$$
\tilde{q}(z) \frac{B(z)}{z} x=\sum_{j=0}^{n} \sum_{k=1}^{j} \hat{q}_{j}\left(B_{k} x\right) z^{n+k-j-1},
$$

which is a polynomial of degree $\leq n-1$, hence $B(z)=\frac{1}{\tilde{q}(z)} P(z)$, where $P$ is an operator polynomial of degree $\leq n$. Thus, degree $B \leq n$.

Next we show that $\operatorname{dim} \mathcal{R}_{B}<\infty$. By Lemma 5.3 the assumption that $\mathcal{R}_{B}=\mathcal{E}$ implies that $T$ does not have an isometric direct summand, hence by Theorem 3.4 we have $\operatorname{dim} \operatorname{ker} T^{*}=\operatorname{rank}\left(I-P_{\mathcal{N}}\right) T P_{\mathcal{N}}$, where $P_{\mathcal{N}}$ denotes the finite rank projection onto $\mathcal{N}$. Since $\mathcal{R}_{B}=\operatorname{ker} T^{*}$ we conclude that $\mathcal{R}_{B}$ is finite dimensional.

Now suppose $\operatorname{dim} \mathcal{R}_{B}<\infty$ and $B$ is rational with degree $\mathrm{B}=n<$ $\infty$, i.e. there is a scalar polynomial $q$ of degree $\leq n$ and an operator 
polynomial $P$ of degree $\leq n$ such that $P(z)=\tilde{q}(z) B(z)$. Then $P(0)=$ 0 . Let $P_{0}(z)=P(z) / z, B_{0}(z)=B(z) / z$. Then for all $x \in \mathcal{D}$ we have

$$
q(L) B_{0} x=L^{n} P_{0} x=0 .
$$

Thus by Lemma 5.1 ran $\Delta \subseteq \operatorname{ker} q(L)$, and hence $\mathcal{N} \subseteq \operatorname{ker} q(L)$. Since $q$ has degree $\leq n$, there is a $k \leq n$ and $\lambda_{1}, \ldots, \lambda_{k} \subseteq \mathbb{D}$ such that $q(z)=q_{1}(z) \prod_{j=1}^{k}\left(z-\lambda_{j}\right)$, where $q_{1}$ is a polynomial without zeros in $\mathbb{D}$. Since $\left\|L^{m} h\right\|_{H^{2}(\mathcal{E})} \rightarrow 0$ for every $h \in H^{2}(\mathcal{E}), L$ cannot have any eigenvalues in $\mathbb{C} \backslash \mathbb{D}$, hence ker $q_{1}(L)=(0)$. Furthermore, since $M_{z}$ does not have any eigenvalues the operator $\left(I-\lambda_{j} M_{z}\right)$ is 1-1 for each $j$, thus the identity $\prod_{j=1}^{k}\left(L-\lambda_{j}\right)=L^{k} \prod_{j=1}^{k}\left(I-\lambda_{j} M_{z}\right)$ shows that

$$
\begin{aligned}
\operatorname{dim} \mathcal{N} & \leq \operatorname{dim} \operatorname{ker} q(L) \leq \operatorname{dim} \operatorname{ker} L^{k} \\
& =k \operatorname{dim} \operatorname{ker} L \leq n \operatorname{dim} \mathcal{E}=n \operatorname{dim} \mathcal{R}_{B} .
\end{aligned}
$$

Now Theorem 1.3 follows form Theorems 3.4, 4.6 and 6.2.

\section{Rational Row Schur functions}

We will now restrict attention to the situation where $K_{w}^{B}(z)$ is a reproducing kernel for a nonzero space of scalar-valued functions, $\mathcal{H}(B) \subseteq$ $H^{2}$. Then $u_{w}(z)=B(z) B(w)^{*}$ is scalar-valued and it is the reproducing kernel for a Hilbert space of analytic functions $\mathcal{H}(u)$. If $\left\{b_{i}\right\}$ is any orthonormal basis of $\mathcal{H}(u)$, then $B(z) B(w)^{*}=\sum_{i \geq 1} b_{i}(z) \overline{b_{i}(w)}$. If we assume that $T=\left(M_{z}, \mathcal{H}(B)\right)$ is bounded, then the dimension of $\mathcal{H}(u)$ equals the rank of $\Delta=T^{*} T-I$, see equation (5.1) of the proof of Lemma 5.1. As we will be interested in the situation where $\Delta$ has finite rank, we can assume that $B=\left(b_{1}, \ldots, b_{k}\right)$ for some linearly independent set of functions $b_{1}, \ldots, b_{k}$. By the theorem of Aleman and Malman (Theorem 1.6) in order to assure that $\left(M_{z}, \mathcal{H}(B)\right)$ be bounded we will need to assume that $1-\sum_{i=1}^{k}\left|b_{i}\right|^{2}$ is log-integrable. Then there will be a unique outer function $a$ such that $a(0)>0$ and $|a|^{2}+\sum_{i=1}^{k}\left|b_{i}\right|^{2}=1$ a.e. on the unit circle. We say that $a$ is the mate of $B$.

Now assume that $B$ is rational of degree $n$, then $a$ will be rational of degree $\leq n$. Indeed, if there are polynomials $q, p_{1}, \ldots, p_{k}$ of degree $\leq n$ such that $b_{i}=p_{i} / q$ and if $1-\sum_{i=1}^{k}\left|b_{i}\right|^{2}$ is log-integrable, then $|q|^{2}-\sum_{i=1}^{k}\left|p_{i}\right|^{2}>0$ a.e. on $\mathbb{T}$, and hence by the Fejér-Riesz Theorem [35], there is a polynomial $p$ of degree $\leq n$ with no zeros in $\mathbb{D}$ and such that $|q|^{2}-\sum_{i=1}^{k}\left|p_{i}\right|^{2}=|p|^{2}$ on the unit circle. Then $a=p / q$ is the required outer function. We note that the denominator of $a$ can be chosen to be the same as the common denominator of the $b_{i}^{\prime} s$. 
Theorem 3.4 implies that under this hypothesis we have $\operatorname{dim} \operatorname{ker} T^{*}=1$, and Theorem 6.2 tells us that

$$
\mathcal{N}=[\operatorname{ran} \Delta]_{T^{*}}=[\operatorname{ran} \Delta]_{L} \text { has dimension } n<\infty .
$$

The generalized eigenspaces of $L$ are all of the form $\operatorname{ker}(L-\lambda)^{n}=$ $\left\{\sum_{j=1}^{n} \frac{a_{j}}{(1-\lambda z)^{j}}: a_{j} \in \mathbb{C}\right\}, 0<|\lambda|<1$, and ker $L^{n}=\left\{\sum_{j=0}^{n-1} a_{j} z^{j}: a_{j} \in\right.$ $\mathbb{C}\}$. That is well-known to be true for the backward shift on $H^{2}$, hence it now follows from the fact that all the functions in the generalized eigenspaces are contained in $\mathcal{H}(B) \subseteq H^{2}$. That means that there are $\alpha_{1}, \ldots, \alpha_{n} \in \mathbb{D}$ such that $\mathcal{N}$ is of the form

$$
\mathcal{N}=\left\{\frac{p(z)}{\prod_{j=1}^{n}\left(1-\alpha_{j} z\right)}: p \text { is a polynomial of degree }<n\right\} .
$$

In fact, in Theorem 6.2 we saw that if $B(0)=0$, then $\prod_{j=1}^{n}\left(1-\alpha_{j} z\right)$ is a constant multiple of the lowest common denominator of the $b_{i}$ 's.

Lemma 7.1. Let $B=\left(b_{1}, \ldots, b_{k}\right)$ be rational functions such that $\log \left(1-\sum_{i}\left|b_{i}\right|^{2}\right) \in L^{1}(\mathbb{T})$ and $B(0)=0$, but $B \neq 0$, then all eigenspaces of $M_{z}^{*}$ are one dimensional.

This implies that the minimal and characteristic polynomials of $M_{z}^{*} \mid \mathcal{N}$ coincide.

Proof. By a theorem of Aleman and Malman ( [7, Theorem 5.5) the polynomials are dense in $\mathcal{H}(B)$, thus the operator $M_{z}$ has a cyclic vector. The lemma follows, because it is well-known that if $T$ is any Hilbert space operator with a cyclic vector $x_{0}$, then every eigenspace is one dimensional. Indeed, for $w \in \mathbb{C}$ we have that the set $\left\{x_{0},(T-\right.$ $\left.w) x_{0},(T-w)^{2} x_{0}, \ldots\right\}$ spans $\mathcal{H}$, and hence $\overline{\operatorname{ran}(T-w)}$ has codimension at most one.

Theorem 7.2. Let $B=\left(b_{1}, \ldots, b_{k}\right)$ be rational functions such that $\log \left(1-\sum_{i}\left|b_{i}\right|^{2}\right) \in L^{1}(\mathbb{T})$ and $B(0)=0$, but $B \neq 0$. Let $T=$ $\left(M_{z}, \mathcal{H}(B)\right)$ and $\mathcal{N}=[\operatorname{ran} \Delta]_{T^{*}}=[\operatorname{ran} \Delta]_{L}$.

If $a$ is the mate of $B$, then

$$
a(z)=a(0) \frac{p\left(\frac{1}{z}\right)}{q\left(\frac{1}{z}\right)}
$$

where $p$ is the characteristic polynomial of $T^{*} \mid \mathcal{N}$ and $q$ is the characteristic polynomial of $L \mid \mathcal{N}$.

Proof. Assume that the degree of $B$ is $n$. Then by Theorem 6.2 the dimension of $\mathcal{N}$ equals $n$. Let $p(z)=\prod_{j=1}^{n}\left(z-\overline{\lambda_{j}}\right)$ be the characteristic polynomial of $T^{*} \mid \mathcal{N}$ and $q(z)=\prod_{j=1}^{n}\left(z-\alpha_{j}\right)$ be the characteristic 
polynomial of $L \mid \mathcal{N}$. Here $\lambda_{1}, \ldots, \lambda_{n} \in \overline{\mathbb{D}}$ and $\alpha_{1}, \ldots, \alpha_{n} \in \mathbb{D}$. Then $\mathcal{N}$ has the form as in (7.1).

Write $\mathcal{M}=\mathcal{N}^{\perp}$. Then since $\operatorname{dim} \mathcal{M}^{\perp}<\infty$ the subspace $\mathcal{M} \ominus$ $z \mathcal{M}$ is 1-dimensional (see e.g. [10], Lemma 2.1) and $M_{z} \mid \mathcal{M}$ is unitarily equivalent to the unilateral shift of multiplicity 1 . Let $\varphi \in \mathcal{M} \ominus z \mathcal{M}$ be a unit vector, then $\left\{z^{n} \varphi\right\}$ forms an orthonormal basis for $\mathcal{M}$ and hence $P_{\mathcal{M}} K_{w}^{B}(z)=\frac{\varphi(z) \overline{\varphi(w)}}{1-z \bar{w}}$. Since $K_{w}^{B}(z)=P_{\mathcal{N}} K_{w}^{B}(z)+P_{\mathcal{M}} K_{w}^{B}(z)$ we conclude that

$$
1-\sum_{i=1}^{k}\left|b_{i}(z)\right|^{2}=\left(1-|z|^{2}\right)\left\|P_{\mathcal{N}} K_{z}^{B}\right\|^{2}+|\varphi(z)|^{2}
$$

for all $z \in \mathbb{D}$. Since all functions in $\mathcal{N}$ are bounded and $\mathcal{N}$ is finite dimensional we let $|z| \rightarrow 1$ and obtain that $|\varphi|^{2}=|a|^{2}$ on $\mathbb{T}$.

Let $C=\left(I-P_{\mathcal{N}}\right) M_{z} P_{\mathcal{N}}$. From Theorem 3.4 we know that rank $C=$ $\operatorname{dim} \operatorname{ker} M_{z}^{*}=1$ and we know $\operatorname{ran} C \subseteq \mathcal{M} \ominus z \mathcal{M}=\operatorname{ker}\left(M_{z} \mid \mathcal{M}\right)^{*}$ since $M_{z}$ expands the norm (see e.g. the proof of Theorem [3.4). Hence there is $f_{1} \in \mathcal{N}$ such that $C f_{1}=\varphi$. Then there is $f_{2} \in \mathcal{N}$ such that $\varphi=z f_{1}-f_{2}$. Considering the form of the functions in $\mathcal{N}$ (see [7.1), we conclude that $\varphi(z)=\frac{h(z)}{\prod_{j=1}^{n}\left(1-\alpha_{j} z\right)}$ for some polynomial $h$ of degree $\leq n$.

Thus, $\varphi \in H^{\infty}$ and in fact $\varphi f \in \mathcal{M}$ for all $f \in \mathcal{H}(B)$. In particular, if $g \in \mathcal{N}$, then $h g \in \mathcal{M}$ or $P_{\mathcal{N}} h\left(M_{z}\right) \mid \mathcal{N}=0$. Let $\tilde{h}(z)=\overline{h(\bar{z})}$, then $\tilde{h}\left(M_{z}^{*}\right) \mid \mathcal{N}=0$. This implies that the minimal polynomial of $M_{z}^{*} \mid \mathcal{N}$ divides $\tilde{h}$. But by Lemma 7.1 the minimal polynomial equals the characteristic polynomial and it has degree $n$. Hence $\tilde{h}$ must be a multiple of $p$, and that implies that $\varphi(z)=\gamma \frac{\prod_{j=1}^{n}\left(z-\lambda_{j}\right)}{\prod_{j=1}^{n}\left(1-\alpha_{j} z\right)}$ for some $\gamma \in \mathbb{C}$. Now $a$ is outer, but has the same modulus as $\varphi$ on $\mathbb{T}$, hence $a(z)=a(0) \frac{\prod_{j=1}^{n}\left(1-\overline{\lambda_{j}} z\right)}{\prod_{j=1}^{n}\left(1-\alpha_{j} z\right)}$. This proves the theorem.

Theorem 1.2 now follows from Theorems 6.2 and 7.2 .

Remark 7.3. Note the relationship between the functions a and $\varphi$ in the proof of Theorem [7.2: $\varphi=S a$ for some finite Blaschke product $S$ and the complex conjugates of the zeros of $S$ must be in $\sigma\left(T^{*} \mid \mathcal{N}\right)$. Thus, if $\sigma\left(T^{*} \mid \mathcal{N}\right) \subseteq \mathbb{T}$, then $\operatorname{deg} a=\operatorname{deg} B=\operatorname{dim} \mathcal{N}$ (since there is no cancellation of linear factors of $\tilde{p}$ and $\tilde{q})$, and $\varphi$ must be a constant multiple of a. 


\section{EXPANSIVE $m$-ISOMETRIES, GENERAL CONSIDERATIONS}

Recall from the Introduction that a bounded linear operator $T$ on a Hilbert space $\mathcal{H}$ is an $m$-isometry for some positive integer $m$ if

$$
\beta_{m}(T)=\left.\sum_{k=0}^{m}(-1)^{m-k}\left(\begin{array}{c}
m \\
k
\end{array}\right) y^{k} x^{k}\right|_{y=T^{*}, x=T}=\sum_{k=0}^{m}(-1)^{m-k}\left(\begin{array}{c}
m \\
k
\end{array}\right) T^{* k} T^{k}=0 .
$$

The first main result of this section will be a theorem that will imply one of the directions of Theorem 1.4 (b). We start with a lemma.

Lemma 8.1. Let $n \in \mathbb{N}, w \in \mathbb{T}, T, P \in \mathcal{B}(\mathcal{H})$, with $P \geq 0$. Then

$$
\sum_{k=0}^{n-1}\left(\begin{array}{c}
n-1 \\
k
\end{array}\right)(-1)^{n-1-k} T^{* k} P T^{k}=\sum_{k=0}^{n-1}\left(\begin{array}{c}
n-1 \\
k
\end{array}\right)\left(T^{*}-\bar{w}\right)^{k} P T^{k}(\bar{w} T-I)^{n-1-k} .
$$

Proof. The intuition for this formula comes from the following application of Agler's hereditary functional calculus:

$$
\begin{aligned}
\left.(y x-1)^{n-1}(P)\right|_{y=T^{*}, x=T} & =\left.[(y-\bar{w}) x+(\bar{w} x-1)]^{n-1}(P)\right|_{y=T^{*}, x=T} \\
& =\sum_{k=0}^{n-1}\left(\begin{array}{c}
n-1 \\
k
\end{array}\right)\left(T^{*}-\bar{w}\right)^{k} P T^{k}(\bar{w} T-1)^{n-1-k} .
\end{aligned}
$$

But it can also be proved by induction. For $n \in \mathbb{N}$ set

$$
\gamma_{n}(P)=\sum_{k=0}^{n-1}\left(\begin{array}{c}
n-1 \\
k
\end{array}\right)(-1)^{n-1-k} T^{* k} P T^{k}
$$

One easily checks that in case $n=1$. Thus, assume that the formula holds for some $n \geq 1$. Then

$$
\begin{aligned}
\gamma_{n+1}(P) & =\sum_{k=0}^{n}\left(\begin{array}{l}
n \\
k
\end{array}\right)(-1)^{n-k} T^{* k} P T^{k} \\
& =T^{*} \gamma_{n}(P) T-\gamma_{n}(P) \\
& =\left(T^{*}-\bar{w}\right) \gamma_{n}(P) T+\gamma_{n}(P)(\bar{w} T-I) .
\end{aligned}
$$

At this point we leave the easy remaining details to the reader.

Theorem 8.2. Let $m \in \mathbb{N}$ and let $T \in \mathcal{B}(\mathcal{H})$ be such that $\Delta=T^{*} T-$ $I \geq 0$.

If there are $w_{1}, w_{2}, \cdots \in \mathbb{T}$ and positive operators $\Delta_{1}, \Delta_{2}, \ldots$ such that $\Delta=\sum_{i \geq 1} \Delta_{i}$ and $\left(T^{*}-\bar{w}_{j}\right)^{m} \Delta_{j}=0$ for each $j \geq 1$, then $T$ is a $2 m$-isometry. 
Proof. Note that the sum converges in the strong operator topology. We use Lemma 8.1 with $w_{i}$ and $P=\Delta_{i}$ for each $i$, and we obtain

$$
\begin{aligned}
\beta_{2 m}(T) & =\sum_{j=0}^{2 m-1}\left(\begin{array}{c}
2 m-1 \\
j
\end{array}\right)(-1)^{2 m-1-j} T^{* j} \Delta T^{j} \\
& =\sum_{i \geq 1} \sum_{j=0}^{2 m-1}\left(\begin{array}{c}
2 m-1 \\
j
\end{array}\right)(-1)^{2 m-1-j} T^{* j} \Delta_{i} T^{j} \\
& =\sum_{i \geq 1} \sum_{j=0}^{2 m-1}\left(\begin{array}{c}
2 m-1 \\
j
\end{array}\right)\left(T^{*}-\overline{w_{i}}\right)^{j} \Delta_{i} T^{j}\left(\bar{w}_{i} T-1\right)^{2 m-1-j} \\
& =0
\end{aligned}
$$

since either $j \geq m$ or $2 m-1-j \geq m$. Hence $T$ is $2 m$-isometry.

Next we will see that for expansive $m$-isometries the finiteness of $\operatorname{dim}[\text { ran } \Delta]_{T^{*}}$ follows from $\Delta$ having finite rank.

Lemma 8.3. If $T$ is an m-isometry, if $\Delta=T^{*} T-I \geq 0$, and if $\Delta$ has finite rank, then

$$
\operatorname{rank} \Delta \leq \operatorname{dim}[\operatorname{ran} \Delta]_{T^{*}} \leq\left\{\begin{array}{ll}
\frac{m}{2} \operatorname{rank} \Delta, & m \text { is even } \\
\frac{m-1}{2} \operatorname{rank} \Delta, & m \text { is odd }
\end{array} .\right.
$$

Proof. The case $m=1$ is trivial, we assume $m \geq 2$. Then

$$
0=\beta_{m}(T)=T^{*} \beta_{m-1}(T) T-\beta_{m-1}(T)=\sum_{j=0}^{m-1}(-1)^{m-1-j}\left(\begin{array}{c}
m-1 \\
j
\end{array}\right) T^{* j} \Delta T^{j} .
$$

Thus we have

$$
\sum_{j \text { even }}^{m-1}\left(\begin{array}{c}
m-1 \\
j
\end{array}\right) T^{* j} \Delta T^{j}=\sum_{j \text { odd }}^{m-1}\left(\begin{array}{c}
m-1 \\
j
\end{array}\right) T^{* j} \Delta T^{j}
$$

Hence we have

$$
\bigvee_{j \text { even }}^{m-1} \operatorname{ran} T^{* j} \Delta T^{j}=\bigvee_{j \text { odd }}^{m-1} \operatorname{ran} T^{* j} \Delta T^{j}
$$

So if $\Delta=\sum_{k=1}^{n} f_{k} \otimes f_{k}$, then when $m=2 l$,

$$
\bigvee_{i=1}^{n}\left\{T^{*} f_{i}, T^{* 3} f_{i} \cdots, T^{*(2 l-1)} f_{i}\right\} \subseteq \bigvee_{i=1}^{n}\left\{f_{i}, T^{* 2} f_{i} \cdots, T^{*(2 l-2)} f_{i}\right\},
$$


and when $m=2 l+1$,

$$
\bigvee_{i=1}^{n}\left\{f_{i}, T^{* 2} f_{i} \cdots, T^{*(2 l)} f_{i}\right\} \subseteq \bigvee_{i=1}^{n}\left\{T^{*} f_{i}, T^{* 3} f_{i} \cdots, T^{*(2 l-1)} f_{i}\right\} .
$$

This implies that the sets on the right hand side of the above two inclusions are $T^{*}$-invariant and hence

$$
\operatorname{dim}[\operatorname{ran} \Delta]_{T^{*}} \leq\left\{\begin{array}{ll}
\frac{m}{2} \operatorname{rank} \Delta, & m \text { is even } \\
\frac{m-1}{2} \operatorname{rank} \Delta, & m \text { is odd }
\end{array} .\right.
$$

The following construction will be crucial for the rest of this section.

Lemma 8.4. Let $T \in \mathcal{B}(\mathcal{H})$ be such that $\Delta=T^{*} T-I \geq 0$. Set $\mathcal{N}=[\operatorname{ran} \Delta]_{T^{*}}$ and assume that $\operatorname{dim} \mathcal{N}=n<\infty$.

If $c=\left(c_{0}, \ldots, c_{n-1}\right)$, where $c_{j}>0$ for each $j, 0 \leq j \leq n-1$, then

$$
\|x\|_{c}^{2}=\sum_{j=0}^{n-1} c_{j}\left\langle\Delta T^{j} x, T^{j} x\right\rangle
$$

defines a Hilbert space norm on $\mathcal{N}$.

Proof. Fix a $c$ as in the hypothesis of the lemma and write $B_{c}=$ $\sum_{j=0}^{n-1} c_{j} T^{* j} \Delta T^{j}$. Thus, $B_{c} \geq 0$ and $\|x\|_{c}^{2}=\left\langle B_{c} x, x\right\rangle$. In order to show that $\|\cdot\|_{c}$ is a norm on $\mathcal{N}$ it suffices to show that $\mathcal{N} \cap \operatorname{ker} B_{c}=(0)$. Let $\Delta=\sum_{i=1}^{N} f_{i} \otimes f_{i}$. Then

$$
\operatorname{ran} B_{c}=\bigvee_{i=1}^{N} \bigvee_{j=0}^{n-1} T^{* j} f_{i}
$$

Note that since $\operatorname{dim} \mathcal{N}=n$ we have that for each fixed $i$ the vector $T^{* n} f_{i}$ must be a linear combination of $f_{i}, T^{*} f_{i}, \ldots, T^{* n-1} f_{i}$. This means that $\bigvee_{j=0}^{n-1} T^{* j} f_{i}$ is invariant for $T^{*}$, and it follows that ran $B_{c}$ is $T^{*}$ invariant. Since ran $B_{c}$ contains $\bigvee_{i=1}^{N}\left\{f_{i}\right\}=\operatorname{ran} \Delta$ we conclude that $\mathcal{N} \subseteq \operatorname{ran} B_{c}$. This implies $\mathcal{N} \cap \operatorname{ker} B_{c}=(0)$.

If $T$ is an operator as in the previous lemma, and if $c_{0}, \ldots, c_{n-1}>0$, then we will write $\mathcal{N}_{c}=\left(\mathcal{N},\|\cdot\|_{c}\right)$ and $A_{c}$ for the operator $A=P_{\mathcal{N}} T \mid \mathcal{N}$ as it acts on $\mathcal{N}_{c}$.

Theorem 8.5. Let $N \in \mathbb{N}, N \geq 2$, and $T \in \mathcal{B}(\mathcal{H})$ be such that $\Delta=$ $T^{*} T-I \geq 0$. Set $\mathcal{N}=[\operatorname{ran} \Delta]_{T^{*}}$ and assume that $\operatorname{dim} \mathcal{N}=n<\infty$.

Then $T$ is an $N$-isometry, if and only if $A_{c}$ is an $(N-1)$-isometry for all $c=\left(c_{0}, \ldots, c_{n-1}\right), c_{j}>0$. 
Proof. Write $D=\Delta^{1 / 2}$, and recall from the proof of Lemma 8.3 that

$$
\beta_{N}(T)=\sum_{k=0}^{N-1}(-1)^{k}\left(\begin{array}{c}
N-1 \\
k
\end{array}\right) T^{* k} \Delta T^{k} .
$$

Thus, for all $x \in \mathcal{H}$ we have

$$
\left\langle\beta_{N}(T) x, x\right\rangle=\sum_{k=0}^{N-1}(-1)^{k}\left(\begin{array}{c}
N-1 \\
k
\end{array}\right)\left\|D T^{k} x\right\|^{2} .
$$

We will use this identity repeatedly.

Fix $c=\left(c_{0}, \ldots, c_{n-1}\right)$ as in the lemma, then for $x \in \mathcal{N}$

$$
\left\|A^{k} x\right\|_{c}^{2}=\sum_{j=0}^{n-1} c_{j}\left\|D T^{j+k} x\right\|^{2}
$$

since $A$ is the compression of $T$ to the semi-invariant subspace $\mathcal{N}$ and $D=D P_{\mathcal{N}}$. This implies that

$$
\begin{aligned}
\sum_{k=0}^{N-1}(-1)^{k}\left(\begin{array}{c}
N-1 \\
k
\end{array}\right)\left\|A^{k} x\right\|_{c}^{2} & =\sum_{j=0}^{n-1} c_{j} \sum_{k=0}^{N-1}(-1)^{k}\left(\begin{array}{c}
N-1 \\
k
\end{array}\right)\left\|D T^{j+k} x\right\|^{2} \\
& =\sum_{j=0}^{n-1} c_{j}\left\langle\beta_{N}(T) T^{j} x, T^{j} x\right\rangle
\end{aligned}
$$

Hence if $T$ is an $N$-isometry, then $A_{c}$ is an $N-1$-isometry on $\mathcal{N}_{c}$ for all tuples $c$ of positive reals.

Conversely, if $A_{c}$ is an $N$-1-isometry on $\mathcal{N}_{c}$ for all positive tuples $c$, then

$$
\sum_{j=0}^{n-1} c_{j}\left\langle\beta_{N}(T) T^{j} x, T^{j} x\right\rangle=0
$$

for all $x \in \mathcal{N}$ and all such $c^{\prime}$ 's. That can only hold, if $\left\langle\beta_{N}(T) x, x\right\rangle=0$ for all $x \in \mathcal{N}$. Thus

$$
\sum_{k=0}^{N-1}(-1)^{k}\left(\begin{array}{c}
N-1 \\
k
\end{array}\right)\left\|D T^{k} x\right\|^{2}=0
$$

for all $x \in \mathcal{N}$.

If $x \in \mathcal{H}$, then $x=x_{1}+x_{2}$ with $x_{1} \in \mathcal{N}$ and $x_{2} \in \mathcal{N}^{\perp}$. Since $\mathcal{N}^{\perp}$ is $T$-invariant we have $D T^{k} x_{2}=0$ for each $k$ and we conclude that $\beta_{N}(T)=0$, i.e. $T$ is an $N$-isometry.

We now show Theorem 1.4 (a). 
Corollary 8.6. Let $m \in \mathbb{N}$, and $T \in \mathcal{B}(\mathcal{H})$ such that $\Delta=T^{*} T-I \geq 0$ and has finite rank.

If $T$ is a $2 m+1$-isometry, then it is a $2 m$-isometry and $\sigma\left(T^{*} \mid \mathcal{N}\right) \subseteq \mathbb{T}$, where $\mathcal{N}=[\operatorname{ran} \Delta]_{T^{*}}$.

Proof. We assume that $T$ is a norm expansive $2 m+1$-isometry such that $\Delta$ has finite rank, and as before we write $A=P_{\mathcal{N}} T \mid \mathcal{N}$. Then by Lemma 8.3 we have $\operatorname{dim} \mathcal{N}<\infty$. Thus, by Theorem 8.5 we conclude that $A_{c}$ is a $2 m$-isometry for each tuple $c$ of positive reals. Agler and Stankus showed that the spectrum of any $n$-isometry is either the closed

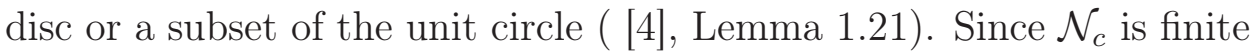
dimensional, this implies that $\sigma(A) \subseteq \mathbb{T}$. Hence $A$ is invertible, and then Proposition 1.23 of [4 implies that $A$ must be a $2 m-1$-isometry in the norm $\|\cdot\|_{c}$. This is true for all tuples $c$ of positive reals, hence another application of Theorem 8.5 shows that $T$ is a $2 m$-isometry.

We saw that $\sigma(A) \subseteq \mathbb{T}$, hence $\sigma\left(T^{*} \mid \mathcal{N}\right)=\sigma\left(A^{*}\right) \subseteq \mathbb{T}$.

For $A \in \mathcal{B}(\mathcal{N})$ and $w \in \mathbb{C}$ let

$$
\mathcal{N}_{w}=\mathcal{N}_{w}(A)=\bigvee_{n \geq 0} \operatorname{ker}(A-w)^{n},
$$

so that for $w \in \sigma_{p}(A)$ the space $\mathcal{N}_{w}$ is the root subspace of $A$ corresponding to the eigenvector $w$, and if $\mathcal{N}$ is finite dimensional, then it is clear that $\mathcal{N}=\bigvee_{w \in \sigma_{p}(A)} \mathcal{N}_{w}$.

We will need the following result, which is a special case of [5], Lemma 19, also see 24], Proposition 6.3, or [14], Theorem 2.7.

Theorem 8.7. Let $\mathcal{N}$ be a finite dimensional Hilbert space, let $A \in$ $\mathcal{B}(\mathcal{N})$ with $\sigma(A)=\left\{w_{1}, \ldots, w_{n}\right\}$, then $A$ is a $2 m-1$-isometry, if and only if

(i) $\sigma(A) \subseteq \mathbb{T}$,

(ii) $\mathcal{N}=\bigoplus_{k=1}^{n} \mathcal{N}_{w_{k}}$,

(iii) $m \geq \min \left\{i: \operatorname{ker}(A-w)^{i}=\mathcal{N}_{w}\right\}$ for each $w \in \sigma(A)$.

If $A$ is a $2 m-1$-isometry, then it is a strict $2 m-1$-isometry, if and only if $m=\max _{w \in \sigma(A)} \min \left\{i: \operatorname{ker}(A-w)^{i}=\mathcal{N}_{w}\right\}$.

The first main step of the converse of Theorem 8.2 if $\Delta$ has finite rank now follows easily.

Theorem 8.8. Let $m \in \mathbb{N}$, and let $T \in \mathcal{B}(\mathcal{H})$ such that $\Delta=T^{*} T-I$ is positive and has finite rank. Let $\mathcal{N}=[\operatorname{ran} \Delta]_{T^{*}}$ and $A=P_{\mathcal{N}} T \mid \mathcal{N}$.

If $T$ is a strict $2 m$-isometry, then $\Delta \mathcal{N}_{w}(A) \perp \mathcal{N}_{z}(A)$ for all $w \neq z$, and $m=\max _{w \in \sigma(A)} \min \left\{i: \operatorname{ker}(A-w)^{i}=\mathcal{N}_{w}\right\}$.

Furthermore, $\Delta^{1 / 2} x \neq 0$ for each nonzero eigenvector $x$ of $A$. 
Remarks: 1. The last sentence of the theorem combined with $\Delta \mathcal{N}_{w}(A) \perp \mathcal{N}_{z}(A)$ for all $z \neq w$ implies that if $T$ is a $2 m$-isometry, then card $\sigma(A) \leq \operatorname{rank} \Delta$.

2. If $\operatorname{dim} \mathcal{N}<\infty$ and $\sigma(A)=\left\{w_{1}, \ldots, w_{k}\right\}$, then let $p(z)=$ $\prod_{j=1}^{k}\left(z-w_{j}\right)^{m_{j}}$ be the minimal polynomial of $A$. Then the condition $m=\max _{w \in \sigma(A)} \min \left\{i: \operatorname{ker}(A-w)^{i}=\mathcal{N}_{w}\right\}$ is easily seen to be equivalent to $m=\max \left\{m_{j}: j=1, \ldots, k\right\}$.

In particular, if $\operatorname{dim} \operatorname{ker} T^{*}=1$, then the condition turns out to be equivalent to $m=\max _{w \in \sigma(A)} \operatorname{dim} \mathcal{N}_{w}$. Indeed, since $\operatorname{dim} \mathcal{N}<\infty$, Theorem 1.3 implies that $T=V \oplus R$, where $V$ is isometric and $R$ is unitarily equivalent to $\left(M_{z}, \mathcal{H}(B)\right)$ for some rational $B \in \mathcal{S}\left(\mathbb{C}^{k}, \mathbb{C}\right)$. Then it follows from Lemma 7.1 that the minimal and characteristic polynomials of $A^{*}$ agree. Then the same is true for $A$ and $\operatorname{dim} \mathcal{N}_{w}(A)=$ $\min \left\{i: \operatorname{ker}(A-w)^{i}=\mathcal{N}_{w}\right\}$ for all $w \in \sigma(A)$.

Proof. Since $T$ is a strict $2 m$-isometry Lemma 8.3 implies that the space $\mathcal{N}$ is finite dimensional. Let $\operatorname{dim} \mathcal{N}=N$. As in Theorem 8.5 we consider tuples $c=\left(c_{0}, c_{1}, \ldots, c_{N-1}\right)$ with $c_{j}>0$ for $j=0, \ldots, N-1$, and we consider the Hilbert space $\mathcal{N}_{c}$ which equals $\mathcal{N}$, but with the norm $\|\cdot\|_{c}$. We write $A_{c}$ to denote the Hilbert space operator $A$ acting in $\mathcal{N}_{c}$. According to Theorem 8.5 $A_{c}$ is a $2 m-1$-isometry for each such $c$. Furthermore, if $A_{c}$ was a $2 m-2$-isometry for every $c$, then by Theorem $8.5 T$ would be a $2 m-1$ isometry. Thus, since $T$ is a strict $2 m$-isometry, there is a tuple $\tilde{c}$ such that the operator $A_{\tilde{c}}$ is a strict $2 m-1$-isometry.

Write $\sigma(A)=\left\{w_{1}, \ldots, w_{n}\right\}$. Then $\sigma\left(A_{c}\right)=\sigma(A)$ and $A_{c}$ and $A$ are similar for all $c$ and all $1 \leq i \leq n$, hence the condition that $m=\max _{w \in \sigma(A)} \min \left\{i: \operatorname{ker}(A-w)^{i}=\mathcal{N}_{w}\right\}$ follows immediately from Theorem 8.7. Condition (ii) of Theorem 8.7 implies that for each $c$ we have $\mathcal{N}_{w_{k}} \perp \mathcal{N}_{w_{j}}$ with respect to $\langle\cdot, \cdot\rangle_{c}$ whenever $j \neq k$. Thus, if $j \neq k$ and if $x \in \mathcal{N}_{w_{j}}, y \in \mathcal{N}_{w_{k}}$, then

$$
\sum_{i=0}^{N-1} c_{i}\left\langle\Delta T^{i} x, T^{i} y\right\rangle=0 .
$$

The spaces $\mathcal{N}_{w}$ do not depend on the $c_{i}$ 's, hence we conclude that each term in the sum had to be 0 . In particular, $\langle\Delta x, y\rangle=0$. This shows $\Delta \mathcal{N}_{w}(A) \perp \mathcal{N}_{z}(A)$ for all $w \neq z$.

If $x \in \mathcal{N}$ is an eigenvector for $A$, say $A x=\lambda x$ for some $\lambda \in \mathbb{C}$, then $|\lambda|^{2 i}\left\|\Delta^{1 / 2} x\right\|^{2}=\left\|\Delta^{1 / 2} A^{i} x\right\|^{2}=\left\|\Delta^{1 / 2} T^{i} x\right\|^{2}$. Hence for any $c$ as above we have $\|x\|_{c}^{2}=\sum_{i=0}^{2 m} c_{i}|\lambda|^{2 i}\left\|\Delta^{1 / 2} x\right\|^{2}$. Since $\|\cdot\|_{c}$ is a norm on $\mathcal{N}$ we conclude that if $x \neq 0$, then $\Delta^{1 / 2} x \neq 0$. 
With the help of a lemma from linear algebra we will transform the condition of the previous theorem into an equivalent condition that will be easy to apply later. Let $T \in \mathcal{B}(\mathcal{H})$, then if $f$ is analytic in a neighborhood of $\sigma(T)$ the Riesz Dunford functional calculus is defined by

$$
f(T)=\frac{1}{2 \pi i} \int_{\gamma} f(z)(z-T)^{-1} d z,
$$

where $\gamma$ is a curve that surrounds $\sigma(T)$ once in the positive direction. If $\tilde{f}(z)=\overline{f(\bar{z})}$, then it is well-known that $f(T)^{*}=\tilde{f}\left(T^{*}\right)$.

If $A$ is an $n \times n$ matrix with $\sigma(A)=\left\{w_{1}, \ldots, w_{k}\right\}$, then let $f_{i}$ be 1 in a neighborhood of $w_{i}$ and 0 in an open set that includes all $w_{j}$ with $j \neq i$. Then $f_{i}(A)$ satisfies $f_{i}(A)^{2}=f_{i}(A)$, it has range equal to $\mathcal{N}_{w_{i}}(A)$, and $\sum_{i=1}^{k} f_{i}(A)=I$. Also note that $\tilde{f}_{i}$ is 1 in a neighborhood of $\bar{w}_{i}$ and 0 elsewhere, so $f_{i}(A)^{*}=\tilde{f}\left(A^{*}\right)$ is an idempotent with range $\mathcal{N}_{\bar{w}_{i}}\left(A^{*}\right)$.

Lemma 8.9. Let $A$ be an $n \times n$ matrix with $\sigma(A)=\left\{w_{1}, \ldots, w_{k}\right\}$ and let $\Delta$ be a positive definite $n \times n$ matrix.

Then $\Delta \mathcal{N}_{w_{i}}(A) \perp \mathcal{N}_{w_{j}}(A)$ for all $i \neq j$, if and only if

$$
\Delta=\sum_{j=1}^{k} \Delta_{j}
$$

where $\Delta_{j} \geq 0$ and $\operatorname{ran} \Delta_{j} \subseteq \mathcal{N}_{\bar{w}_{j}}\left(A^{*}\right)$ for each $j=1, \ldots, k$.

Note that if the conditions of the lemma are satisfied, then the geometry of the spaces $\mathcal{N}_{\bar{w}_{j}}\left(A^{*}\right)$ implies that $\operatorname{dim} \mathcal{N}=\sum_{j=1}^{k} \operatorname{dim} \mathcal{N}_{\bar{w}_{j}}\left(A^{*}\right)$ and rank $\Delta=\sum_{j=1}^{k} \operatorname{rank} \Delta_{j}$.

Proof. Note that $f_{i}(A) f_{j}(A)=0$ and $\tilde{f}_{i}\left(A^{*}\right) \tilde{f}_{j}\left(A^{*}\right)=0$ for all $i \neq j$.

First suppose that $\Delta \mathcal{N}_{w_{i}}(A) \perp \mathcal{N}_{w_{j}}(A)$ for all $i \neq j$. Then for all $i \neq j$ we have $\tilde{f}_{j}\left(A^{*}\right) \Delta f_{i}(A)=0$. Hence

$$
\Delta=\left(\sum_{j=1}^{k} \tilde{f}_{j}\left(A^{*}\right)\right) \Delta\left(\sum_{i=1}^{k} f_{i}(A)\right)=\sum_{j=1}^{k} \tilde{f}_{j}\left(A^{*}\right) \Delta f_{j}(A) .
$$

Set $\Delta_{j}=\tilde{f}_{j}\left(A^{*}\right) \Delta f_{j}(A)=f_{j}(A)^{*} \Delta f_{j}(A)$. Then $\Delta_{j} \geq 0, \Delta=\sum_{j=1}^{k} \Delta_{j}$ and $\operatorname{ran} \Delta_{j} \subseteq \operatorname{ran} \tilde{f}_{j}\left(A^{*}\right)=\mathcal{N}_{\bar{w}_{j}}\left(A^{*}\right)$.

Conversely, assume that $\Delta=\sum_{j=1}^{k} \Delta_{j}$ for nonnegative matrices $\Delta_{j}$ that satisfy $\operatorname{ran} \Delta_{j} \subseteq \mathcal{N}_{\bar{w}_{j}}\left(A^{*}\right)$ for each $j=1, \ldots, k$. Let $i \neq j$ and let $x \in \mathcal{N}_{w_{i}}(A), y \in \mathcal{N}_{w_{j}}(A)$. Then $x=f_{i}(A) x$ and $y=f_{j}(A) y$, and 
$\Delta_{m}=\tilde{f}_{m}\left(A^{*}\right) \Delta_{m}$ for each $m$, hence

$$
\begin{aligned}
\langle\Delta x, y\rangle & =\sum_{m=1}^{k}\left\langle\tilde{f}_{m}\left(A^{*}\right) \Delta_{m} x, f_{j}(A) y\right\rangle \\
& =\sum_{m=1}^{k}\left\langle\Delta_{m} x, f_{m}(A) f_{j}(A) y\right\rangle \\
& =\left\langle\Delta_{j} x, y\right\rangle \\
& =\left\langle x, \Delta_{j} y\right\rangle \\
& =\left\langle f_{i}(A) x, \tilde{f}_{j}\left(A^{*}\right) \Delta_{j} y\right\rangle \\
& =\left\langle f_{j}(A) f_{i}(A) x, y\right\rangle \\
& =0
\end{aligned}
$$

Hence $\Delta \mathcal{N}_{w_{i}}(A) \perp \mathcal{N}_{w_{j}}(A)$ for all $i \neq j$.

Finally we can prove the remaining direction of Theorem 1.4 (b).

Theorem 8.10. Let $T \in \mathcal{B}(\mathcal{H})$ be a $2 m$-isometry such that $\Delta=T^{*} T-$ $I$ is positive and has finite rank.

Then there are $w_{1}, \ldots, w_{k} \in \mathbb{T}$ and positive operators $\Delta_{1}, \ldots, \Delta_{k}$ such that $\Delta=\sum_{j=1}^{k} \Delta_{j}$ and $\left(T^{*}-\bar{w}_{j}\right)^{m} \Delta_{j}=0$ for each $j=1, \ldots, k$.

If $p(z)=\prod_{j=1}^{k}\left(z-\bar{w}_{j}\right)^{m_{j}}$ is the minimal polynomial of $T^{*} \mid \mathcal{N}$, then $T$ is a strict $2 m_{0}$-isometry, where $m_{0}=\max \left\{m_{j}: j=1, \ldots, k\right\}$.

Proof. This theorem follows from Theorem 8.8, Lemma 8.9, and the observation that $T^{*} \mid \mathcal{N}=A^{*}$, where $A=P_{\mathcal{N}} T \mid \mathcal{N}$.

Corollary 8.11. Let $m \in \mathbb{N}, T \in \mathcal{B}(\mathcal{H})$ be such that $\Delta=T^{*} T-I=$ $\eta \otimes \eta$. Let $\mathcal{N}=[\eta]_{T^{*}}$ Then the following are equivalent:

(a) $T$ is a strict $2 m$-isometry,

(b) $\operatorname{dim} \mathcal{N}=m$ and there exists $w \in \mathbb{T}$ such that $\left(T^{*}-\bar{w}\right)^{m} \eta=0$,

(c) there exists $w \in \mathbb{T}$ such that $p(z)=(z-\bar{w})^{m}$ is the characteristic polynomial of $T^{*} \mid \mathcal{N}$.

Proof. Since $\eta$ is a cyclic vector for $T^{*} \mid \mathcal{N}$ it is clear that (b) and (c) are equivalent, and that the characteristic polynomial of $T^{*} \mid \mathcal{N}$ equals its minimal polynomial. Thus, if (b) holds then Theorems 1.4 or 8.10 ensure that $T$ is a strict $2 m$-isometry, i.e. condition (b) implies condition (a).

In order to prove $(\mathrm{a}) \Rightarrow(\mathrm{b})$ we suppose that $T$ is a strict $2 m$-isometry. Then Theorem 1.4 implies that there exists $w \in \mathbb{T}$ such that $\left(T^{*}-\right.$ $\bar{w})^{m} \eta=0$. This implies that $\operatorname{dim} \mathcal{N} \leq m$. If $\operatorname{dim} \mathcal{N}<m$, then the minimal polynomial of $T^{*} \mid \mathcal{N}$ is not $(z-\bar{w})^{m}$, which in turn implies that $T$ is not a strict $2 m$-isometry. Hence $\operatorname{dim} \mathcal{N}=m$. 
9. ExpAnsive $2 m$-ISOMETRIES, rank $\Delta=1$

Let $m \in \mathbb{N}$. If $w \in \mathbb{T}$, then we define the local Dirichlet space of order $m$ at $w$ by

$\mathcal{D}_{w}^{m}=\left\{p+(z-w)^{m} g: p\right.$ is a polynomial of degree $<m$ and $\left.g \in H^{2}\right\}$.

One easily checks that if $f \in \mathcal{D}_{w}^{m}$, then the polynomial $p$ of degree $<m$ and the function $g \in H^{2}$ such that $f=p+(z-w)^{m} g$ are unique. Thus, if $f \in H^{2}$, then $f \in \mathcal{D}_{w}^{m}$ if and only if there is a (unique) polynomial $p$ of degree $<m$ such that $g=(f-p) /(z-w)^{m} \in H^{2}$. We define the local Dirichlet integral of order $m$ of $f$ at $w$ by

$$
D_{w}^{m}(f)=\inf \left\{\left\|\frac{f-p}{(z-w)^{m}}\right\|_{H^{2}}^{2}: p \text { is a polynomial of degree }<m\right\} .
$$

It is clear that if $f \in \mathcal{D}_{w}^{m}$ extends to be analytic in a neighborhood of $w$, then

$$
D_{w}^{m}(f)=\int_{|z|=1}\left|\frac{f(z)-T_{m-1}(f, w)(z)}{(z-w)^{m}}\right|^{2} \frac{|d z|}{2 \pi},
$$

where $T_{m-1}(f, w)$ be the $(m-1)$-th order Taylor polynomial of $f$ at $w$,

$$
T_{m-1}(f, w)(z)=\sum_{j=0}^{m-1} \frac{f^{(j)}(w)}{j !}(z-w)^{j} .
$$

Lemma 9.1. Let $m \in \mathbb{N}, w \in \mathbb{T}$, and $f \in H^{2}$, then $f \in \mathcal{D}_{w}^{m}$, if and only if for each $j=0, \ldots, m-1$ the function $f^{(j)}$ has nontangential limit $b_{j}$ at $w$, and $D_{w}^{m}(f)$ as defined in (9.1) with $T_{m-1}(f, w)(z)=$ $\sum_{j=0}^{m-1} \frac{b_{j}}{j !}(z-w)^{j}$ is finite.

Proof. Note that $(z-w)^{m}$ is an outer function. Thus, if $f \in H^{2}$ and if $p$ is any polynomial, then $(f-p) /(z-w)^{m}$ is in the Smirnov class, and hence it will be in $H^{2}$, whenever it is in $L^{2}(\mathbb{T})$.

If $f \in \mathcal{D}_{w}^{m}$, then $f=p+(z-w)^{m} g$ for some $g \in H^{2}$ and a polynomial $p$ of degree $<m$. Let $i$ be an integer with $0 \leq i \leq m-1$. Then since $g \in H^{2}$, we have

Thus,

$$
g^{(i)}(z)=\left\langle g, \frac{\partial^{i} k_{z}^{H^{2}}}{\partial \bar{z}^{i}}\right\rangle_{H^{2}}
$$

Then in the region

$$
\left|g^{(i)}(z)\right| \precsim \frac{1}{(1-|z|)^{i+1 / 2}}
$$

$$
\Gamma_{\alpha}(w)=\{z \in \mathbb{D}:|z-w|<\alpha(1-|z|)\}
$$


the function $\left((z-w)^{m} g\right)^{(j)}(z), 0 \leq j \leq m-1$, goes to zero as $z \rightarrow w$. Thus, for each $j=0, \ldots, m-1$ the function $f^{(j)}$ has nontangential limit equal to $b_{j}=p^{(j)}(w)$ at $w$. Hence $p(z)=\sum_{j=0}^{m-1} \frac{b_{j}}{j !}(z-w)^{j}=$ $T_{m-1}(f, w)(z)$.

If $f \in \mathcal{D}_{w}^{m}$, then for $0 \leq j \leq m-1$ we will write $f^{(j)}(w)$ to denote the limit of $f^{(j)}(z)$ as $z \rightarrow w$ nontangentially. We define a norm on $\mathcal{D}_{w}^{m}$ by

$$
\|f\|^{2}=\|f\|_{H^{2}}^{2}+D_{w}^{m}(f) .
$$

We note that if $m=1$, then we obtain the local Dirichlet integral $D_{w}^{1}(f)=D_{w}(f)$ that was introduced in [34].

Lemma 9.2. Let $m \in \mathbb{N}, w \in \mathbb{T}$. Then for each $0 \leq j \leq m-1$ the functional $f \rightarrow f^{(j)}(w)$ is bounded on $\mathcal{D}_{w}^{m}$.

Proof. Note that by Lemma 9.1 we have $\sup \left\{\left|f^{(j)}(z)\right|: z \in \Gamma_{\alpha}(w)\right\}<$ $\infty$ for every $f \in \mathcal{D}_{w}^{m}$. Thus, the lemma follows from the uniform boundedness principle and Lemma 9.1.

Lemma 9.3. Let $f \in H^{2}$. Then $f \in \mathcal{D}_{w}^{m}$ if and only if $z f \in \mathcal{D}_{w}^{m}$. Furthermore, if $g \in H^{2}$ such that for $z \in \mathbb{D}$ we have

$$
f(z)-(z-w)^{m} g(z)=\sum_{j=0}^{m-1} a_{j}(z-w)^{j},
$$

then

$$
D_{w}^{m}(z f)=D_{w}^{m}(f)+\left|a_{m-1}\right|^{2} .
$$

Proof. If $f=\sum_{j=0}^{m-1} a_{j}(z-w)^{j}+(z-w)^{m} g$, then

$$
z f=a_{0} w+\sum_{j=1}^{m-1}\left(a_{j-1}+a_{j} w\right)(z-w)^{j}+(z-w)^{m}\left(a_{m-1}+z g\right) .
$$

This implies $D_{w}^{m}(z f)=\left\|a_{m-1}+z g\right\|_{H^{2}}^{2}=\left|a_{m-1}\right|^{2}+D_{w}^{m}(f)$. Since by Lemma 9.2 the functional $f \rightarrow a_{m-1}$ is bounded on $\mathcal{D}_{w}^{m}$, it shows that $M_{z}$ is bounded on $\mathcal{D}_{w}^{m}$ and that it expands the norm. Similarly, one sees that $z f \in \mathcal{D}_{w}^{m}$ implies $f \in \mathcal{D}_{w}^{m}$.

It follows that $T=\left(M_{z}, \mathcal{D}_{w}^{m}\right)$ is bounded, norm-expansive, analytic, and satisfies $\operatorname{dim} \operatorname{ker} T^{*}=\operatorname{rank}\left(T^{*} T-I\right)=1$, see Lemma 2.1 of [31]. Thus, by Theorem 4.6 $T$ is unitarily equivalent to $\left(M_{z}, \mathcal{H}(b)\right)$ for some non-extremal $b$ in the unit ball of $H^{\infty}$. If we assume that $b(0)=0$, then the constant functions form ker $M_{z}^{*}$ in both cases, and hence we must have $\mathcal{H}(b)=\mathcal{D}_{w}^{m}$ with equality of norms. It will follow from the next theorem that $b(z)=z^{m} / q(z)$, where $q$ is a polynomial of degree 
$m$ that has no zeros in $\mathbb{D}$ and such that $|q(z)|^{2}=1+|z-w|^{2 m}$ for all $z \in \mathbb{T}$. The following is a version of Theorem 1.1 .

Theorem 9.4. Let $b$ be non-extremal in the unit ball with $b(0)=0$, and let $m \in \mathbb{N}$. Then the following are equivalent:

a) $\left(M_{z}, \mathcal{H}(b)\right)$ is a strict $2 m$-isometry,

b) $b$ is rational of degree $\leq m$ and there is $w \in \mathbb{T}$ and a polynomial $q$ of degree $\leq m$ with no zeros in $\overline{\mathbb{D}}$ and such that the mate a of $b$ is of the form $a(z)=\frac{(z-w)^{m}}{q(z)}$.

c) there is $w \in \mathbb{T}$ and a polynomial $p$ of degree $<m$ such that $p(w) \neq 0$ and

$$
\|f\|_{\mathcal{H}(b)}^{2}=\|f\|_{H^{2}}^{2}+D_{w}^{m}(p f) .
$$

In fact, if $\left(M_{z}, \mathcal{H}(b)\right)$ is a strict $2 m$-isometry, then there is $w \in \mathbb{T}$ and there are polynomials $p$ and $q$ of degree $\leq m$ such that

(i) $|q(z)|^{2}=|p(z)|^{2}+|z-w|^{2 m}$ for all $z \in \mathbb{T}$,

(ii) $p(w) \neq 0, p(0)=0$

(iii) $q(z) \neq 0$ for all $z \in \overline{\mathbb{D}}$,

(iv) $b=p / q$,

(v) $\|f\|_{\mathcal{H}(b)}^{2}=\|f\|_{H^{2}}^{2}+D_{w}^{m}(\tilde{p} f)$, where $\tilde{p}(z)=z^{m} \overline{p\left(\frac{1}{\bar{z}}\right)}$.

Note that $p(0)=0$ implies that degree $\tilde{p} \leq m-1$.

Proof. The conditions $(i)-(v)$ will be verified while we are showing the equivalence of the conditions (a), (b) and (c). We start by proving the equivalence of (a) and (b). Set $T=\left(M_{z}, \mathcal{H}(b)\right), \Delta=T^{*} T-I$, and $\mathcal{N}=[\text { ran } \Delta]_{T^{*}}$. Then $T$ is analytic, norm expansive, and satisfies $\operatorname{dim} \operatorname{ker} T^{*}=\operatorname{rank} \Delta=1$. Thus, by Corollary $8.11 T$ is a strict $2 m$ isometry, if and only if the characteristic polynomial of $T^{*} \mid \mathcal{N}$ equals $(z-\bar{w})^{m}$ for some $w \in \mathbb{T}$.

Thus, the equivalence of (a) and (b) now follows from Theorem 1.2.

Next we prove the equivalence of (b) and (c). We start by assuming condition (b) is satisfied. Then we may assume that $b=p / q$ for some polynomials $p$ and $q$ of degree $\leq m$, and that the mate $a$ of $b$ is of the form $a(z)=(z-w)^{m} / q(z)$ for some $w \in \mathbb{T}$. The polynomial $q$ has no zeros in the closed unit disc, and $|p(z)|^{2}+|z-w|^{2 m}=|q(z)|^{2}$ for all $z \in \mathbb{T}$. This implies $p(w) \neq 0$.

For $u \in H^{\infty}$ we will write $T_{u}$ for the multiplication operator on $H^{2}$, $T_{u} f=u f$ for all $f \in H^{2}$.

Now let $f \in \mathcal{H}(b)$. The norm of $f$ in $\mathcal{H}(b)$ is given by

$$
\|f\|_{\mathcal{H}(b)}^{2}=\|f\|_{H^{2}}^{2}+\left\|f^{+}\right\|_{H^{2}}^{2}
$$


where $f^{+}$is the unique function that satisfies $T_{b}^{*} f=T_{a}^{*} f^{+}$, see [38]. Since $q$ has no zeros in the closed disc, this identity is equivalent to $T_{p}^{*} f=T_{(z-w)^{m}}^{*} f^{+}$. Thus, if $S$ denotes the unilateral shift, then $p(S)^{*} f-$ $(S-w)^{m^{*}} f^{+}=0$.

Note that

$$
p(S)^{*} f=\sum_{k=0}^{m} \overline{\hat{p}(k)} S^{* k} f=S^{* m} \sum_{k=0}^{m} \overline{\hat{p}(k)} z^{m-k} f=S^{* m}(\tilde{p} f),
$$

where as before $\tilde{p}(z)=z^{m} \overline{p\left(\frac{1}{\bar{z}}\right)}$. Similarly, $(S-w)^{m^{*}} f^{+}=S^{* m}((1-$ $\left.\bar{w} z)^{m} f^{+}\right)$and hence $\tilde{p} f-(1-\bar{w} z)^{m} f^{+} \in \operatorname{ker} S^{* m}$. This implies that there is a polynomial $R$ of degree $<m$ such that

$$
f^{+}=\frac{\tilde{p} f-R}{(z-w)^{m}} \in H^{2} .
$$

Thus $\|f\|_{\mathcal{H}(b)}^{2}=\|f\|_{H^{2}}^{2}+D_{w}^{m}(\tilde{p} f)$. The hypothesis that $b(0)=0$ implies that $p(0)=0$ and that implies that the degree of $\tilde{p}$ is $<m$. Note that since $|w|=1$ we have $p(w)=0$, if and only if $\tilde{p}(w)=0$, and we had already noted that $p(w) \neq 0$. This proves $(b) \Rightarrow(c)$.

Finally suppose that (c) holds. Then for some $w \in \mathbb{T}$ and some polynomial $p$ of degree $<m$ with $p(w) \neq 0$ we have $D_{w}^{m}(p f)=\left\|f^{+}\right\|_{H^{2}}^{2}$ for every $f \in \mathcal{H}(b)$. Here $f^{+}$is the unique $H^{2}$-function with $T_{b}^{*} f=$ $T_{a}^{*} f^{+}$. The definition of the local Dirichlet integral of order $m$ at $w$ implies that there must be a polynomial $R$ of degree $<m$ such that $(z-w)^{m} f^{+}=p f-R$. This implies that $S^{* m}\left(p f-(z-w)^{m} f^{+}\right)=0$. Calculating as above, we see that this is equivalent to

$$
T_{\tilde{p}}^{*} f=\tilde{p}(S)^{*} f=(1-\bar{w} S)^{m *} f^{+}=(-1)^{m} w^{m} T_{(z-w)^{m}}^{*} f^{+} .
$$

In this case $\tilde{p}(z)=z^{m} \overline{p(1 / \bar{z})}$, so that the fact that degree $p<m$ implies that $\tilde{p}(0)=0$. Now by the Fejér-Riesz Theorem there is a polynomial $q$ of degree $\leq m$ such that $|z-w|^{2 m}+|\tilde{p}(z)|^{2}=|q(z)|^{2}$ for all $z \in \mathbb{T}$. Since $p(w) \neq 0$ we have $\tilde{p}(w) \neq 0$ and hence we may assume that $q$ has no zeros in the closed unit disc. Then the adjoint Toeplitz operator $T_{q}^{*}$ is invertible. Now set $\tilde{b}(z)=\tilde{p}(z) / q(z)$, then by the properties of $q$ there must be a $c \in \mathbb{T}$ such that $\tilde{a}(z)=c(z-w)^{m} / q(z)$ is the mate of $\tilde{b}$. The invertibility of $T_{q}^{*}$ implies that $T_{\tilde{b}}^{*} f=(-1)^{m} w^{m} c T_{\tilde{a}}^{*} f^{+}$. This implies that $\|f\|_{\mathcal{H}(\tilde{b})}^{2}=\|f\|_{H^{2}}^{2}+\left\|f^{+}\right\|_{H^{2}}^{2}=\|f\|_{\mathcal{H}(b)}^{2}$ for all $f \in \mathcal{H}(b)$. Thus $b(z)=c^{\prime} \tilde{b}(z)$ for some constant $c^{\prime} \in \mathbb{T}$, see Lemma 4.8. Hence $b$ is a rational function of degree $\leq m$ and its mate is of the form $(z-w)^{m} / q(z)$ for some polynomial $q$ that has no zeros in the closed unit disc. Hence $(c) \Rightarrow(b)$. 
Corollary 9.5. Let $m \in \mathbb{N}, w \in \mathbb{T}$ and $p$ be a polynomial of degree $\leq m-1$ with $p(w) \neq 0$, and let $b$ be in the unit ball of $H^{\infty}$ such that $\|f\|_{\mathcal{H}(b)}^{2}=\|f\|_{H^{2}}^{2}+D_{w}^{m}(p f)$ for all $f \in \mathcal{H}(b)$.

Then $\mathcal{H}(b)=\mathcal{D}_{w}^{m}$ with equivalence of norms, i.e. there are constants $c, C>0$ such that

$$
c\left(\|f\|_{H^{2}}^{2}+D_{w}^{m}(f)\right) \leq\|f\|_{H^{2}}^{2}+D_{w}^{m}(p f) \leq C\left(\|f\|_{H^{2}}^{2}+D_{w}^{m}(f)\right) .
$$

Proof. We already showed in Lemma 9.3 that $M_{z}$ acts boundedly on $\mathcal{D}_{w}^{m}$, hence it is clear that $D_{w}^{m}(p f) \lesssim D_{w}^{m}(f)$ and hence $\mathcal{D}_{w}^{m} \subseteq \mathcal{H}(b)$. Thus, in order to complete the proof it will suffice to show that $\mathcal{H}(b) \subseteq$ $D_{w}^{m}=\mathcal{P}_{m-1} \dot{+}(z-w)^{m} H^{2}$. The norm inequality will then follow by a routine application of the Closed Graph Theorem.

Let $T=\left(M_{z}, \mathcal{H}(b)\right), \Delta=T^{*} T-I, \mathcal{N}=[\operatorname{ran} \Delta]_{T^{*}}$, and $\mathcal{M}=$ $\mathcal{H}(b) \ominus \mathcal{N}$. In the proof of Theorem 7.2 we showed that $\mathcal{H}(b)=\varphi H^{2} \oplus \mathcal{N}$ for $\varphi \in \mathcal{M} \ominus z \mathcal{M},\|\varphi\|=1$, and by Remark 7.3 we have $\varphi=e^{i t} a$. Thus, by Theorem 9.4 and equation 7.1

$$
\mathcal{H}(b)=\frac{(z-w)^{m}}{q} H^{2} \oplus \frac{1}{q} \mathcal{P}_{m-1},
$$

where $q$ is a polynomial with no zeros in $\overline{\mathbb{D}}$. The result follows since multiplication by $q$ is an invertible operator on $\mathcal{H}(b)$, see [38], Section IV-5.

\section{Finite RAnK EXPansive $2 m$-ISOMEtRIES}

In this Section we will establish Theorem 1.5.

We start with a formula for the norm in $\mathcal{H}(B)$, which in the current form is due to [7]. Closely related formulas were also used in [8], [9], and [6]. In fact, with hindsight this formula can be used to motivate the definition of the first order local Dirichlet integral.

Lemma 10.1. Let $B=\left(b_{1}, \ldots, b_{n}\right) \in \mathcal{S}\left(\mathbb{C}^{n}, \mathbb{C}\right)$ with $B(0)=0$ and such that $T=\left(M_{z}, \mathcal{H}(B)\right)$ is bounded. Set $\Delta=T^{*} T-I$ and $D=\Delta^{1 / 2}$. Then for all polynomials $g \in \mathcal{H}(B)$ we have

$$
\|g\|_{\mathcal{H}(B)}^{2}=\|g\|_{H^{2}}^{2}+\int_{|\lambda|=1}\left\|D \frac{g-g(\lambda)}{z-\lambda}\right\|_{\mathcal{H}(B)}^{2} \frac{|d \lambda|}{2 \pi} .
$$

Proof. It is clear that $\mathcal{H}(B)$ satisfies the conditions (A1')-(A3') of Aleman and Malman's paper [7. Hence their Proposition 2.5 applies, i.e.

$\|g\|_{\mathcal{H}(B)}^{2}=\|g\|_{H^{2}}^{2}+\lim _{r \rightarrow 1} \int_{|\lambda|=1}\left[\left\|z \frac{g-g(r \lambda)}{z-r \lambda}\right\|_{\mathcal{H}(B)}^{2}-r^{2}\left\|\frac{g-g(r \lambda)}{z-r \lambda}\right\|_{\mathcal{H}(B)}^{2}\right] \frac{|d \lambda|}{2 \pi}$.

Thus, for polynomials $g$ this lemma follows from the dominated convergence theorem. 
Lemma 10.2. Let $m \in \mathbb{N}, w \in \mathbb{T}$, and $p$ be a polynomial of degree $\leq m-1$. Then

$$
D_{w}^{m}(p f)=\int_{|\lambda|=1}\left[D_{w}^{m}\left(z p \frac{f-f(\lambda)}{z-\lambda}\right)-D_{w}^{m}\left(p \frac{f-f(\lambda)}{z-\lambda}\right)\right] \frac{|d \lambda|}{2 \pi}
$$

for every polynomial $f$.

Proof. First assume $p(w) \neq 0$ and define a norm on the polynomials by $\|f\|^{2}=\|f\|_{H^{2}}^{2}+D_{w}^{m}(p f)$. Then by Theorem $9.4 M_{z}$ extends to define a bounded $2 m$-isometry $T=\left(M_{z}, \mathcal{H}\right)$ on some space $\mathcal{H}=\mathcal{H}(b)$. Thus, in this case the lemma follows from Lemma 10.1 .

If $p$ is identically 0 , then the lemma is trivial. If $p \neq 0$, but $p(w)=0$, then $p(z)=(z-w)^{k} p_{1}(z)$ for some polynomial $p_{1}$ with $p_{1}(w) \neq 0$. Now we can apply the formula $D_{w}^{m}\left((z-w)^{k} p_{1} f\right)=D_{w}^{m-k}\left(p_{1} f\right)$ and apply the first case.

Lemma 10.3. Let $m \in \mathbb{N}, w \in \mathbb{T}, B \in \mathcal{S}\left(\mathbb{C}^{n}, \mathbb{C}\right)$ be such that $B(0)=0$ and $T=\left(M_{z}, \mathcal{H}(B)\right)$ is a bounded operator.

If $f_{0} \in \mathcal{H}(B)$ such that $\left(T^{*}-\bar{w}\right)^{m} f_{0}=0$, but $\left(T^{*}-\bar{w}\right)^{m-1} f_{0} \neq 0$, then there is a polynomial $p$ of degree $\leq m-1$, with $p(w) \neq 0$, and such that

$$
D_{w}^{m}(z p g)-D_{w}^{m}(p g)=\left|\left\langle g, f_{0}\right\rangle\right|_{\mathcal{H}(B)}^{2}
$$

for every polynomial $g$.

Furthermore, with that $p$ we have

$$
D_{w}^{m}(p f)=\int_{|\lambda|=1}\left|\left\langle\frac{f-f(\lambda)}{z-\lambda}, f_{0}\right\rangle_{\mathcal{H}(B)}\right|^{2} \frac{|d \lambda|}{2 \pi}
$$

for all polynomials $f$.

Note that if drop the part of the hypothesis that assumes $\left(T^{*}-\right.$ $\bar{w})^{m-1} f_{0} \neq 0$, then one can still deduce the existence of a polynomial $p$ which satisfies all parts of the conclusion except that possibly $p(w)=0$. That is because under that hypothesis there is $m_{0} \leq m$ such that the hypothesis of the lemma is completely satisfied with $m_{0}$, hence there is a polynomial $p_{0}$ of degree $\leq m_{0}-1$ such that the conclusion of the lemma holds for $m_{0}$. Then we can set $p(z)=(z-w)^{m-m_{0}} p_{0}(z)$ and use the identity $D_{w}^{m_{0}}\left(p_{0} f\right)=D_{w}^{m}(p f)$ to deduce the claim.

Proof. First we show that $\left\langle(z-w)^{m-1}, f_{0}\right\rangle_{\mathcal{H}(B)} \neq 0$. The polynomials are dense in $\mathcal{H}(B)$ (see [7]), hence by the hypothesis there is a polynomial $q$ such that $\left\langle q,\left(T^{*}-\bar{w}\right)^{m-1} f_{0}\right\rangle \neq 0$. The function $r(z)=\frac{q(z)-q(w)}{z-w}$ 
is a polynomial and satisfies $q(z)=q(w)+(z-w) r(z)$, hence

$$
\begin{aligned}
0 & \neq\left\langle q,\left(T^{*}-\bar{w}\right)^{m-1} f_{0}\right\rangle \\
& =q(w)\left\langle(z-w)^{m-1}, f_{0}\right\rangle_{\mathcal{H}(B)}+\left\langle(z-w) r,\left(T^{*}-\bar{w}\right)^{m-1} f_{0}\right\rangle \\
& =q(w)\left\langle(z-w)^{m-1}, f_{0}\right\rangle_{\mathcal{H}(B)}+\left\langle r,\left(T^{*}-\bar{w}\right)^{m} f_{0}\right\rangle \\
& =q(w)\left\langle(z-w)^{m-1}, f_{0}\right\rangle_{\mathcal{H}(B)}
\end{aligned}
$$

Hence $\left\langle(z-w)^{m-1}, f_{0}\right\rangle_{\mathcal{H}(B)} \neq 0$.

Now set $p(\lambda)=\sum_{j=0}^{m-1}\left\langle(z-w)^{m-1-j}, f_{0}\right\rangle_{\mathcal{H}(B)}(\lambda-w)^{j}$. Then $p$ is a polynomial of degree $\leq m-1$ and $p(w) \neq 0$.

Let $g$ be a polynomial, then $g(z)=\sum_{j \geq 0} \frac{g^{(j)}(w)}{j !}(z-w)^{j}$ and since $\left\langle(z-w)^{k}, f_{0}\right\rangle_{\mathcal{H}(B)}=0$ for all $k \geq m$ we have

$$
\begin{aligned}
\left\langle g, f_{0}\right\rangle_{\mathcal{H}(B)} & =\sum_{j=0}^{m-1} \frac{g^{(j)}(w)}{j !}\left\langle(z-w)^{j}, f_{0}\right\rangle_{\mathcal{H}(B)} \\
& =\sum_{j=0}^{m-1} \frac{g^{(j)}(w)}{j !} \frac{p^{(m-1-j)}(w)}{(m-1-j) !} \\
& =\frac{(p g)^{(m-1)}(w)}{(m-1) !} .
\end{aligned}
$$

Now Lemma 9.3 implies that $D_{w}^{m}(z p g)-D_{w}^{m}(p g)=\left|\left\langle g, f_{0}\right\rangle_{\mathcal{H}(B)}\right|^{2}$.

Finally, for $|\lambda|=1$ we apply this formula with $g(z)=\frac{f(z)-f(\lambda)}{z-\lambda}$, and integrate over the unit circle with respect to $\frac{|d \lambda|}{2 \pi}$. The lemma then follows from Lemma 10.2 .

Theorem 10.4. Let $m \in \mathbb{N}, B \in \mathcal{S}\left(\mathbb{C}^{n}, \mathbb{C}\right)$ be such that $B(0)=0$ and $T=\left(M_{z}, \mathcal{H}(B)\right)$ is a bounded operator. Write $\Delta=T^{*} T-I$, $\mathcal{N}=[\operatorname{ran} \Delta]_{T^{*}}, A=P_{\mathcal{N}} T \mid \mathcal{N}$, and assume that

- $\operatorname{dim} \mathcal{N}<\infty$

- $\sigma(A)=\left\{w_{1}, \ldots w_{k}\right\}$, and

- $p(z)=\prod_{j=1}^{k}\left(z-w_{j}\right)^{m_{j}}$ is the characteristic polynomial of $A$.

If $T$ is a strict $2 m$-isometry, then

$$
\sigma(A) \subseteq \mathbb{T}, \quad m=\max \left\{m_{j}: 1 \leq j \leq k\right\},
$$

and there are $n_{1}, \ldots, n_{k} \in \mathbb{N}$ with $n_{j} \leq m_{j}$ for all $j$ and $\sum_{j=1}^{k} n_{j}=$ rank $\Delta$, and there are polynomials $\left\{p_{i j}\right\}_{1 \leq j \leq k, 1 \leq i \leq n_{j}}$ with $p_{1 j}\left(w_{j}\right) \neq 0$ for all $j$, such that the degree of $p_{i j} i s \leq m_{j}-1$ for all $j$ and $i$, and 
such that

$$
\|f\|_{\mathcal{H}(B)}^{2}=\|f\|_{H^{2}}^{2}+\sum_{j=1}^{k} \sum_{i=1}^{n_{j}} D_{w_{j}}^{m_{j}}\left(p_{i j} f\right)
$$

for all $f \in \mathcal{H}(B)$.

Proof. The hypothesis that $\operatorname{dim} \mathcal{N}<\infty$ implies that $B$ is rational (see Theorem 1.2). Since $B \in \mathcal{S}\left(\mathbb{C}^{n}, \mathbb{C}\right)$ we have $\operatorname{dim} \operatorname{ker} T^{*}=1$. Thus, if $T$ is a strict $2 m$-isometry, then $\sigma(A) \subseteq \mathbb{T}$ and $m=\max \left\{m_{j}: 1 \leq\right.$ $j \leq k\}$ by Theorem 8.8 and Remark 2 following it. Also, since $T$ is a $2 m$-isometry, Theorem 8.10 implies that $\Delta=\sum_{j=1}^{k} \Delta_{j}$, where each $\Delta_{j} \geq 0$ and $\operatorname{ran} \Delta_{j} \subseteq \mathcal{N}_{\bar{w}_{j}}\left(A^{*}\right)$. Let $n_{j}=\operatorname{rank} \Delta_{j}$, then $n_{j} \leq$ $\operatorname{dim} \mathcal{N}_{\bar{w}_{j}}\left(A^{*}\right)=m_{j}$. This is because the characteristic polynomial of $A$ equals its minimal polynomial (see Lemma 7.1).

Let $1 \leq j \leq k$. Since ran $\Delta_{j} \subseteq \mathcal{N}_{\bar{w}_{j}}\left(A^{*}\right)$ there are $f_{1 j}, \ldots, f_{n_{j} j} \in$ $\mathcal{N}_{\bar{w}_{j}}\left(A^{*}\right)$ such that $\Delta_{j}=\sum_{i=1}^{n_{j}} f_{i j} \otimes f_{i j}$. For fixed $j$ we have $\left(T^{*}-\right.$ $\left.\bar{w}_{j}\right)^{m_{j}} f_{i j}=0$ for all $i$, and we may assume that $\left(T^{*}-\bar{w}_{j}\right)^{m_{j}-1} f_{1 j} \neq 0$. Indeed, if $\left(T^{*}-\bar{w}_{j}\right)^{m_{j}-1} f_{i j}=0$ for all $i$, then one shows that $\frac{p(z)}{z-w_{j}}$ would annihilate $A$. This contradicts that the minimal and characteristic polynomials must agree. Now we use Lemma 10.3 and the remark immediately following it to deduce that there are polynomials $\left\{p_{i j}\right\}_{1 \leq j \leq k, 1 \leq i \leq n_{j}}$ with $p_{1 j}\left(w_{j}\right) \neq 0$ for all $j$, such that the degree of $p_{i j}$ is $\leq m_{j}-1$ for all $j$ and $i$ and such that for each $j$ and $i$ we have

$$
D_{w_{j}}^{m_{j}}\left(p_{i j} f\right)=\int_{|\lambda|=1}\left|\left\langle\frac{f-f(\lambda)}{z-\lambda}, f_{i j}\right\rangle_{\mathcal{H}(B)}\right|^{2} \frac{|d \lambda|}{2 \pi}
$$

for every polynomial $f$.

Write $D=\Delta^{1 / 2}$. Then since $\Delta=\sum_{j=1}^{k} \sum_{i=1}^{n_{j}} f_{i j} \otimes f_{i j}$ we have for every polynomial $g$ that

$$
\|D g\|_{\mathcal{H}(B)}^{2}=\sum_{j=1}^{k} \sum_{i=1}^{n_{j}}\left|\left\langle g, f_{i j}\right\rangle_{\mathcal{H}(B)}\right|^{2} .
$$

Hence Lemma 10.1 implies

$$
\begin{aligned}
\|f\|_{\mathcal{H}(B)}^{2} & =\|f\|_{H^{2}}^{2}+\sum_{j=1}^{k} \sum_{i=1}^{n_{j}} \int_{|\lambda|=1}\left|\left\langle\frac{f-f(\lambda)}{z-\lambda}, f_{i j}\right\rangle_{\mathcal{H}(B)}\right|^{2} \frac{|d \lambda|}{2 \pi} \\
& =\|f\|_{H^{2}}^{2}+\sum_{j=1}^{k} \sum_{i=1}^{n_{j}} D_{w_{j}}^{m_{j}}\left(p_{i j} f\right)
\end{aligned}
$$

for every polynomial $f$. The theorem follows since the polynomials are dense in $\mathcal{H}(B)$. 
In order to establish the missing parts of the proof of Theorem 1.5 we prove the following converse of Theorem 10.4 .

Theorem 10.5. Let $w_{1}, \ldots, w_{k}$ be mutually distinct points in $\mathbb{T}$, let $m_{1}, \ldots, m_{k}, n_{1}, \ldots n_{k} \in \mathbb{N}$, set $\tilde{n}_{j}=\min \left(m_{j}, n_{j}\right)$, and let $\left\{p_{i j}\right\}_{1 \leq j \leq k, 1 \leq i \leq n_{j}}$ be polynomials such that the degree of $p_{i j}$ is $\leq m_{j}-1$ and such that $p_{1, j}\left(w_{j}\right) \neq 0$ for each $j$.

Then there is a $n \in \mathbb{N}, n \leq \sum_{j=1}^{k} \tilde{n}_{j}$, and a rational $B \in \mathcal{S}\left(\mathbb{C}^{n}, \mathbb{C}\right)$ such that $B(0)=0$ and

$$
\|f\|_{\mathcal{H}(B)}^{2}=\|f\|_{H^{2}}^{2}+\sum_{j=1}^{k} \sum_{i=1}^{n_{j}} D_{w_{j}}^{m_{j}}\left(p_{i j} f\right)
$$

for all $f \in \mathcal{H}(B)$.

Furthermore, if $m=\max \left\{m_{j}: 1 \leq j \leq k\right\}$, then $T=\left(M_{z}, \mathcal{H}(B)\right)$ is an expansive $2 m$-isometry with rank $\Delta \leq n$. If $\mathcal{N}=[\operatorname{ran} \Delta]_{T^{*}}$ and $A=P_{\mathcal{N}} T \mid \mathcal{N}$, then $\sigma(A)=\left\{w_{1}, \ldots w_{k}\right\}$ and the characteristic polynomial of $A$ is $p_{A}(z)=\prod_{j=1}^{k}\left(z-w_{j}\right)^{m_{j}}$. Furthermore, the mate a of $B$ is of the form $a(z)=\frac{p_{A}(z)}{q(z)}$, where $q$ is a polynomial of degree $\leq \sum_{j=1}^{k} m_{j}$ and it has no zeros in the closed unit disc. $q$ is determined by

$$
|q(z)|^{2}=\left|p_{A}(z)\right|^{2}+\sum_{j=1}^{k}\left|\frac{p_{A}(z)}{\left(z-w_{j}\right)^{m_{j}}}\right|^{2} \sum_{i=1}^{n_{j}}\left|p_{i j}(z)\right|^{2} \text { for all }|z|=1 .
$$

Proof. For $1 \leq j \leq k$ and $1 \leq i \leq n_{j}$ define norms by

$$
\begin{gathered}
\|f\|_{i j}^{2}=\|f\|_{H^{2}}^{2}+D_{w_{j}}^{m_{j}}\left(p_{i j} f\right), \\
\|f\|_{j}^{2}=\|f\|_{H^{2}}^{2}+\sum_{i=1}^{n_{j}} D_{w_{j}}^{m_{j}}\left(p_{i j} f\right),
\end{gathered}
$$

and define

$$
\|f\|^{2}=\|f\|_{H^{2}}^{2}+\sum_{j=1}^{k} \sum_{i=1}^{n_{j}} D_{w_{j}}^{m_{j}}\left(p_{i j} f\right) .
$$

Furthermore, let $\mathcal{H}_{i j}, \mathcal{H}_{j}$, and $\mathcal{H}$ be the Hilbert function spaces that consist of all analytic functions such that the corresponding norms are finite. Then by Theorem 9.4 each operator $T_{i j}=\left(M_{z}, \mathcal{H}_{i j}\right)$ is a bounded, analytic, and expansive $2 m_{j}$-isometry with $\operatorname{dim} \operatorname{ker} T_{i j}^{*}=1$. It is then immediately clear that each of the operators $T_{j}=\left(M_{z}, \mathcal{H}_{j}\right)$ and $T=\left(M_{z}, \mathcal{H}\right)$ are bounded, expansive, and analytic. We also note 
that $\mathcal{H}_{j}=\bigcap_{i} \mathcal{H}_{i j}$ and $\mathcal{H}=\bigcap_{j} \mathcal{H}_{j}$. By Lemma 2.1 of [31] the condition $\operatorname{dim} \operatorname{ker} T_{i j}^{*}=1$ is equivalent to $f \in \mathcal{H}_{i j}, f(0)=0 \Rightarrow f(z) / z \in$ $\mathcal{H}_{i j}$. Thus, we may apply that lemma to $T_{j}$ and $T$ and conclude that $\operatorname{dim} \operatorname{ker} T_{j}^{*}=\operatorname{dim} \operatorname{ker} T^{*}=1$ for all $j$. Note that an operator $\left(M_{z}, \mathcal{K}\right)$ is a $2 M$-isometry, if and only if

$$
\sum_{r=0}^{2 M}\left(\begin{array}{c}
2 M \\
r
\end{array}\right)(-1)^{r}\left\|z^{r} f\right\|_{\mathcal{K}}^{2}=0
$$

for all $f \in \mathcal{K}$. Thus, it is clear that for each $j T_{j}$ is a $2 m_{j}$-isometry and that $T$ is a $2 m$-isometry, where $m=\max \left\{m_{j}: 1 \leq j \leq k\right\}$. Furthermore, if $\Delta=T^{*} T-I, \Delta_{j}=T_{j}^{*} T_{j}-I$, then by Lemma 9.3 we have

$$
\langle\Delta f, f\rangle_{\mathcal{H}}=\sum_{j=1}^{k} \sum_{i=1}^{n_{j}}\left|\frac{\left(p_{i j} f\right)^{\left(m_{j}-1\right)}\left(w_{j}\right)}{\left(m_{j}-1\right) !}\right|^{2}
$$

for all $f \in \mathcal{H}$ and

$$
\left\langle\Delta_{j} f, f\right\rangle_{\mathcal{H}_{j}}=\sum_{i=1}^{n_{j}}\left|\frac{\left(p_{i j} f\right)^{\left(m_{j}-1\right)}\left(w_{j}\right)}{\left(m_{j}-1\right) !}\right|^{2}
$$

for all $f \in \mathcal{H}_{j}$. This implies that $\operatorname{rank} \Delta_{j} \leq n_{j}$ and rank $\Delta \leq$ $\sum_{j=1}^{k} n_{j}<\infty$. Thus, there are rational and scalar-valued Schur functions $B_{j}, B$ such that $T_{j}$ is unitarily equivalent to $\left(M_{z}, \mathcal{H}\left(B_{j}\right)\right)$ and $T$ is unitarily equivalent to $\left(M_{z}, \mathcal{H}(B)\right)$. Furthermore, one similarly observes that $\Delta_{j}\left(T_{j}-w_{j}\right)^{m_{j}}=0$, hence by taking the adjoint and using Lemma 7.1 we see that dim ran $\Delta_{j} \leq m_{j}$. Thus, we have rank $\Delta_{j} \leq \tilde{n}_{j}$ for each $j$.

Now for each $j$ let $J_{j}$ the inclusion map of $\mathcal{H} \subseteq \mathcal{H}_{j}$, then $\Delta=$ $\sum_{j=1}^{k} J_{j}^{*} \Delta_{j} J_{j}$. Thus, we must have $n=\operatorname{rank} \Delta \leq \sum_{j=1}^{k} \tilde{n}_{j}$. That means that by Theorem 4.6 we may choose $B \in \mathcal{S}\left(\mathbb{C}^{n}, \mathbb{C}\right)$ such that $B(0)=0$ and $\left(M_{z}, \mathcal{H}(B)\right)$ is unitarily equivalent to $T$. As usual, since $\operatorname{dim} \operatorname{ker} T^{*}=1$, the unitary operator must be given by multiplication by a function $G$, and the condition $B(0)=0$ is equivalent to $\operatorname{ker} M_{z}^{*}=$ $\operatorname{ker} T^{*}$ being equal to the constant functions. Thus, the multiplier $G$ must be constant, and hence $\mathcal{H}=\mathcal{H}(B)$ and $\|f\|_{\mathcal{H}(B)}=\|f\|$ for all $f \in \mathcal{H}$.

Next we use the definitions $\mathcal{N}=[\operatorname{ran} \Delta]_{T^{*}}$ and $A=P_{\mathcal{N}} T \mid \mathcal{N}$, and $p_{A}(z)=\prod_{j=1}^{k}\left(z-w_{j}\right)^{m_{j}}$. We will show that $p_{A}$ is the minimal polynomial of $A$. That will show that $\sigma(A)=\left\{w_{1}, \ldots, w_{k}\right\}$ and that $p_{A}$ is the characteristic polynomial of $A$ (by Lemma 7.1).

Note that if $p$ is any polynomial, then $p(A)=0$, if and only if ran $p(T) \perp T^{* n} \Delta y$ for all $n \geq 0$ and all $y \in \mathcal{H}$. That condition is 
equivalent to $\Delta p(T)=0$. Of course, a similar statement holds for each $T_{j}$.

Fix $1 \leq j \leq k$. The condition $p_{1 j}\left(w_{j}\right) \neq 0$ implies by Theorem 9.4 that $T_{1 j}$ is a strict $2 m_{j}$-isometry, i.e. $\beta_{2 m_{j}-1}\left(T_{1 j}\right) \neq 0$. For each $i$ the fact that $T_{i j}$ is a $2 m_{j}$-isometry, implies that $\beta_{2 m_{j}-1}\left(T_{i j}\right) \geq 0$ (see [4]), hence one easily checks that $\beta_{2 m_{j}-1}\left(T_{j}\right) \neq 0$. This means that $T_{j}$ must be a strict $2 m_{j}$-isometry with $\Delta_{j}\left(T_{j}-w_{j}\right)^{m_{j}}=0$, but $\Delta_{j}\left(T_{j}-w_{j}\right)^{m_{j}-1} \neq 0$ (see Corollary 8.11). Then for any polynomial $r$ we have

$$
\Delta_{j}\left(T_{j}-w_{j}\right)^{m_{j}-1}\left(r\left(T_{j}\right)-r\left(w_{j}\right)\right)=0
$$

and hence if $r\left(w_{j}\right) \neq 0$, then

$$
\Delta_{j}\left(T_{j}-w_{j}\right)^{m_{j}-1} r\left(T_{j}\right) \neq 0 .
$$

Then we have $\Delta p_{A}(T)=\sum_{j=1}^{k} J_{j}^{*} \Delta_{j} p_{A}\left(T_{j}\right) J_{j}=0$ since for each $j$ we have $\Delta_{j}\left(T_{j}-w_{j}\right)^{m_{j}}=0$. Thus, $p_{A}(A)=0$.

For each $j$ we can factor $p_{A}(z)=\left(z-w_{j}\right)^{m_{j}} p_{j}(z)$ for some polynomial $p_{j}$ with $p_{j}\left(w_{j}\right) \neq 0$, but $\Delta_{i} p_{j}\left(T_{i}\right)=0$ for all $i \neq j$. Thus,

$$
\begin{aligned}
\Delta\left(T-w_{j}\right)^{m_{j}-1} p_{j}(T) & =\sum_{i=1}^{k} J_{i}^{*} \Delta_{i}\left(T_{i}-w_{j}\right)^{m_{j}-1} p_{j}\left(T_{i}\right) J_{i} \\
& =J_{j}^{*} \Delta_{j}\left(T_{j}-w_{j}\right)^{m_{j}-1} p_{j}\left(w_{j}\right) J_{j} \neq 0,
\end{aligned}
$$

since $J_{j}$ is $1-1$. This proves that $p_{A}$ is the minimal polynomial of $A$.

As mentioned above we conclude that $p_{A}$ is the characteristic polynomial of $A$, and hence $N=\operatorname{dim} \mathcal{N}=\operatorname{deg} p_{A}=\sum_{j=1}^{k} m_{j}$. Hence by Theorem 1.2 we conclude that the degree of $B$ is $N$. Then Theorem 1.2 also implies that $a(z)=p_{A}(z) / q(z)$ for some polynomial $q$ of degree $\leq N$ and which has no zeros in $\overline{\mathbb{D}}$.

Finally, let $\mathcal{M}=\mathcal{N}^{\perp}$ and let $\varphi \in \mathcal{M} \ominus z \mathcal{M},\|\varphi\|=1$. Then by Remark 7.3 we may assume that $\varphi=a$. Note that since for each $j$ the function $a$ has a zero of multiplicity $m_{j}$ at $w_{j}$ we observe that for each $j$ and $i$ the Taylor polynomial at $w_{j} T_{m_{j}-1}\left(p_{i j} a, w_{j}\right)(z)=0$ for all $z$. This implies that for all $n \in \mathbb{N}$

$$
0=\left\langle z^{n} \varphi, \varphi\right\rangle_{\mathcal{H}(B)}=\int_{|z|=1} z^{n}|a|^{2}\left(1+\sum_{j=1}^{k} \sum_{i=1}^{n_{j}} \frac{\left|p_{i j}\right|^{2}}{\left|z-w_{j}\right|^{2 m_{j}}}\right) \frac{|d z|}{2 \pi} .
$$

Hence

$$
|a(z)|^{-2}=1+\sum_{j=1}^{k} \sum_{i=1}^{n_{j}} \frac{\left|p_{i j}(z)\right|^{2}}{\left|z-w_{j}\right|^{2 m_{j}}}
$$


for a.e. $z \in \mathbb{T}$. Considering that $a=p_{A} / q$ this shows that $q$ satisfies equation (10.1).

This last calculation reverses. If a polynomial $q_{0}$ of degree $\leq N$ satisfies (10.1), then the function $\psi=p_{A} / q_{0}$ satisfies $\left\langle z^{n} \psi, \psi\langle\mathcal{H}(B)=0\right.$ for all $n \in \mathbb{N}$, and it has norm 1 . Since $p_{A}(A)=0$ we must have that $\psi \in \mathcal{M}$. Then since $\mathcal{M}=\varphi H^{2}$ we obtain $\psi=\varphi f$ for some $f \in H^{2}$, and by the orthogonality condition $f$ would have to be an inner function. Using the definitions of $\psi$ and $\varphi=a$ we see that $q_{0}=q / f$, and that implies that $f$ must be a constant of modulus 1 ( $q$ has no zeros in $\mathbb{D})$.

\section{A Construction}

Our Theorem 1.5 about $2 m$-isometries leaves open the question of what the precise connection between the polynomials $p_{i j}$ and the Schur function $B$ is. The general construction that we described in Section 4 is not very explicit in this case. In general there may be many different Schur functions $B$ that have the same mate $a$. If $a$ is of the form $a(z)=(z-w)^{m} / q(z)$, then for any $B$ with mate $a$, with $B(0)=0$, and of degree $m$, it turns out that $T=\left(M_{z}, \mathcal{H}(B)\right)$ is a $2 m$-isometry. In fact, in this case Theorem 1.2 implies that $(z-w)^{m}$ is the characteristic polynomial of $A$, hence $\left(T^{*}-\bar{w}\right)^{m} \Delta=0$, and the conclusion follows from part (b) of Theorem [1.4. However, if $\sigma(A)$ contains more than one point, then it may happen that two Schur functions $B_{1}$ and $B_{2}$ have the same mate $a$, and $B_{1}$ is a $2 m$-isometry, but $B_{2}$ is not.

Suppose all the conditions are met in Theorem 10.5, Then

$$
\|f\|_{\mathcal{H}(B)}^{2}=\|f\|_{H^{2}}^{2}+\sum_{j=1}^{k} \sum_{i=1}^{n_{j}} D_{w_{j}}^{m_{j}}\left(p_{i j} f\right), f \in \mathcal{H}(B) .
$$

$p_{A}(z)=\prod_{j=1}^{k}\left(z-w_{j}\right)^{m_{j}}, a(z)=\frac{p_{A}(z)}{q(z)}$, where $q$ is a polynomial of degree $\leq \sum_{j=1}^{k} m_{j}$ and it has no zeros in the closed unit disc. $q$ is determined by (10.1).

We will now indicate how to calculate the reproducing kernel $K_{w}^{B}(z)$ by use of the Grammian of a dual basis to $\left\{\bar{\partial}^{i} K_{w_{j}}^{B}\right\}_{i j}$. In the case of 2 -isometries this idea was developed in [3].

We know that $K_{w}^{B}(z)=P_{\mathcal{N}} K_{w}^{B}(z)+\frac{a(z) \overline{a(w)}}{1-z \bar{w}}$, where $\mathcal{N}$ is the $\sum_{j=1}^{k} m_{j^{-}}$ dimensional space spanned by the functions $\left\{\bar{\partial}^{i} K_{w_{j}}^{B}=\frac{\partial^{i} K_{w_{j}}^{B}}{\bar{\partial} w^{i}}: 0 \leq i \leq\right.$ $\left.m_{j}-1,1 \leq j \leq k\right\}$. We also know that a dual basis to $\left\{\bar{\partial}^{i} K_{w_{j}}^{B}\right.$ : $\left.0 \leq i \leq m_{j}-1,1 \leq j \leq k\right\}$ must be of the form $\left\{f_{i j}: 0 \leq i \leq\right.$ 
$\left.m_{j}-1,1 \leq j \leq k\right\}$, where $f_{i j}(z)=\frac{a(z)}{\left(z-w_{j}\right)^{m_{j}-i}} g_{i j}$, and $g_{i j}$ are polynomials with $\operatorname{deg} g_{i j} \leq m_{j}-1-i$, and are determined by the conditions that $f_{i j}^{(i)}\left(w_{j}\right)=1, f_{i j}^{(l)}\left(w_{j}\right)=0, i+1 \leq l \leq m_{j}-1$. Indeed, these functions are in $\mathcal{N}$ by (7.1), and by the choice of constants they are dual to the functions $\left\{\bar{\partial}^{i} K_{w_{j}}^{B}\right\}$. We note that the coefficients of the functions $g_{i j}$ can be calculated as solutions to linear equations (that require knowledge of the polynomial $q$ ). Next we form the Grammian matrix

$$
F=\left[\left\langle f_{i j}, f_{s t}\right\rangle_{\mathcal{H}(B)}\right],
$$

where $\left\langle f_{i j}, f_{s t}\right\rangle_{\mathcal{H}(B)}$ can be calculated by (11.1).

Now note that

$$
P_{\mathcal{N}}=\sum_{j=1}^{k} \sum_{i=0}^{m_{j}-1} \bar{\partial}^{i} K_{w_{j}}^{B} \otimes f_{i j}=\sum_{j=1}^{k} \sum_{i=0}^{m_{j}-1} f_{i j} \otimes \bar{\partial}^{i} K_{w_{j}}^{B} .
$$

Thus,

$$
P_{\mathcal{N}} K_{w}^{B}(z)=\sum_{j=1}^{k} \sum_{i=0}^{m_{j}-1} \overline{\bar{\partial}^{i} K_{w_{j}}^{B}(w)} f_{i j}(z) .
$$

Let $\left[f_{i j}\right]^{t}$ be the column vector $\left[f_{i j}\right]_{0 \leq i \leq m_{j}-1,1 \leq j \leq k}^{t}$. Then from

$$
f_{s t}=\sum_{j=1}^{k} \sum_{i=0}^{m_{j}-1}\left\langle f_{s t}, f_{i j}\right\rangle_{\mathcal{H}(B)} \bar{\partial}^{i} K_{w_{j}}^{B}, 0 \leq s \leq m_{t}-1,1 \leq t \leq k,
$$

we obtain that $\left[f_{i j}\right]^{t}=F\left[\bar{\partial}^{i} K_{w_{j}}^{B}\right]^{t}$ and hence $\left[\bar{\partial}^{i} K_{w_{j}}^{B}\right]^{t}=F^{-1}\left[f_{i j}\right]^{t}$.

Now we have determined $f_{i j}$ and $\bar{\partial}^{i} K_{w_{j}}^{B}$. So we obtain the formula for the reproducing kernel $K_{w}^{B}(z)=P_{\mathcal{N}} K_{w}^{B}(z)+\frac{a(z) \overline{a(w)}}{1-z \bar{w}}$.

We use the following example to illustrate the above construction.

Example 11.1. Define a norm 2-isometric norm by

$$
\|f\|^{2}=\|f\|_{H^{2}}^{2}+\frac{9}{16}\left(D_{1}(f)+D_{-1}(f)\right) .
$$

Then $p_{A}(z)=z^{2}-1, a=p_{A} / q$, where for $|z|=1$

$$
|q(z)|^{2}=\left|z^{2}-1\right|^{2}+\frac{9}{16}\left(|z+1|^{2}+|z-1|^{2}\right)=\frac{17}{4}-2 \operatorname{Re} z^{2} .
$$

Thus $q(z)=\frac{z^{2}-4}{2}$, and $a(z)=\frac{2\left(z^{2}-1\right)}{z^{2}-4}$.

Note that no other 2-isometry will have the same $a$. Indeed, the only possible candidates would have to have norm $\|f\|_{*}^{2}=\|f\|_{H^{2}}^{2}+$ $c_{1} D_{1}(f)+c_{2} D_{-1}(f)$, and unless $c_{1}=c_{2}=9 / 16$ that would lead to a 
different $q$. Thus, there is a unique space $\mathcal{H}(B)$ such that $\left(M_{z}, \mathcal{H}(B)\right)$ is a 2 -isometry and $B$ has mate $a$.

The function $b(z)=\frac{3 z}{z^{2}-4}$ satisfies $b(0)=0$, has degree 2 , and has mate equal to $a$, but by Theorem $1.1\left(M_{z}, \mathcal{H}(b)\right)$ cannot be 2-isometric. That is because in our example the rank of $\Delta$ equals 2 . So the reproducing kernel for the given norm has to be of the form $K_{w}^{B}(z)$ for $B=\left(b_{1}, b_{2}\right)$ for linearly independent functions $b_{1}$ and $b_{2}$ with $B(0)=0$ and such that the degree of $B$ is 2 .

Now we use the above construction to find $K_{w}^{B}(z)$. We have $\mathcal{N}$ is the 2-dimensional space spanned by the kernel functions $K_{1}^{B}$ and $K_{-1}^{B}$. Then the dual basis to $\left\{K_{1}^{B}, K_{-1}^{B}\right\}$ is $\left\{f_{1}, f_{2}\right\}$, where $f_{1}(z)=$ $\frac{-3}{2} \frac{z+1}{z^{2}-4}, f_{2}(z)=\frac{3}{2} \frac{z-1}{z^{2}-4}$. By calculation, we obtain

$$
F=\left[\begin{array}{ll}
\left\langle f_{1}, f_{1}\right\rangle & \left\langle f_{1}, f_{2}\right\rangle \\
\left\langle f_{2}, f_{1}\right\rangle & \left\langle f_{2}, f_{2}\right\rangle
\end{array}\right]=\left[\begin{array}{ll}
\frac{21}{32} & \frac{-9}{32} \\
\frac{-9}{32} & \frac{21}{32}
\end{array}\right], \quad F^{-1}=\left[\begin{array}{ll}
\frac{28}{15} & \frac{12}{15} \\
\frac{12}{15} & \frac{28}{15}
\end{array}\right] .
$$

Then

$$
K_{1}^{B}(z)=\frac{-8 / 5 z-4}{z^{2}-4}, \quad K_{-1}^{B}(z)=\frac{8 / 5 z-4}{z^{2}-4} .
$$

So

$$
\begin{aligned}
K_{w}^{B}(z) & =\frac{1-B(z) B(w)^{*}}{1-z \bar{w}}=P_{\mathcal{N}} K_{w}^{B}(z)+\frac{a(z) \overline{a(w)}}{1-z \bar{w}} \\
& =\overline{K_{1}^{B}(w)} f_{1}(z)+\overline{K_{-1}^{B}(w)} f_{2}(z)+\frac{a(z) \overline{a(w)}}{1-z \bar{w}} \\
& =\frac{1-(z \bar{w})(9 / 5 z \bar{w}+36 / 5) /\left(\left(z^{2}-4\right)\left(\bar{w}^{2}-4\right)\right)}{1-z \bar{w}} .
\end{aligned}
$$

There are many choices for $B$ which give the same expression $K_{w}^{B}(z)$, for example, we can take $B=\left(b_{1}, b_{2}\right), b_{1}(z)=\frac{6 / \sqrt{5} z}{z^{2}-4}, b_{2}(z)=\frac{3 / \sqrt{5} z^{2}}{z^{2}-4}$.

Since this example only involves two points 1 and -1 , we have another way to derive the reproducing kernel $K_{w}^{B}(z)$. Suppose $B(z)=$ $\frac{z\left(v_{1} z+v_{2}\right)}{q(z)}, v_{i}=\left(\alpha_{i}, \beta_{i}\right), i=1,2$. Note that $a(z)=\frac{2\left(z^{2}-1\right)}{z^{2}-4}$, and $|B(z)|^{2}+$ $|a(z)|^{2}=1, z \in \mathbb{T}$. We obtain that $\left\langle v_{1}, v_{2}\right\rangle=0,\left\|v_{1}\right\|^{2}+\left\|v_{2}\right\|^{2}=9$. So

$$
\begin{aligned}
K_{w}^{B}(z) & =\frac{1-B(z) B(w)^{*}}{1-z \bar{w}} \\
& =\frac{1-(z \bar{w})\left(\left(9-\left\|v_{2}\right\|^{2}\right) z \bar{w}+\left\|v_{2}\right\|^{2}\right) /\left(\left(z^{2}-4\right)\left(\bar{w}^{2}-4\right)\right)}{1-z \bar{w}} .
\end{aligned}
$$


Then using $1=\left\langle z, \bar{\partial} K_{0}^{B}(z)\right\rangle$, we find $\left\|v_{2}\right\|^{2}=\frac{36}{5}$, and

$$
K_{w}^{B}(z)=\frac{1-(z \bar{w})(9 / 5 z \bar{w}+36 / 5) /\left(\left(z^{2}-4\right)\left(\bar{w}^{2}-4\right)\right)}{1-z \bar{w}} .
$$

\section{REFERENCES}

[1] J. Agler, A disconjugacy theorem for Toeplitz operators. Amer. J. Math. 112, no. 1, 1-14 (1990).

[2] J. Agler, J.E. McCarthy, Pick Interpolation and Hilbert Function Spaces. Graduate Studies in Mathematics, 44. American Mathematical Society, Providence, RI, 2002.

[3] J. Agler, S. Richter, private conversations, early 1990s.

[4] J. Agler, M. Stankus, $m$-isometric transformations of Hilbert space. Part I, Integral Equations Operator Theory 21, no. 4, 383-429 (1995), Part II, Integral Equations Operator Theory 23, no. 1, 1-48 (1995), Part III, Integral Equations Operator Theory 24, no. 4, 379-421 (1996).

[5] J. Agler, J.W. Helton, M. Stankus, Classification of hereditary matrices. Linear Algebra Appl. 274, 125-160 (1998).

[6] A. Aleman, N. Feldman, W. Ross, The Hardy space of a slit domain. Frontiers in Mathematics. Birkhäuser Verlag, Basel, 2009.

[7] A. Aleman, B. Malman, Hilbert spaces of analytic functions with a contractive backward shift. J. Funct. Anal. 277, no. 1, 157-199 (2019).

[8] A. Aleman, S. Richter, Simply invariant subspaces of $H^{2}$ of some multiply connected regions. Integral Equations Operator Theory 24 (1996), no. 2, 127155.

[9] A. Aleman, S. Richter, C. Sundberg, Beurling's theorem for the Bergman space. Acta Math. 177, 275-310 (1996).

[10] A. Aleman, S. Richter, C. Sundberg, The majorization function and the index of invariant subspaces in the Bergman spaces, J. Analyse Math. 86, 139-182 (2002).

[11] A. Baranov, Y. Belov, A. Borichev, Spectral synthesis in de Branges spaces. Geom. Funct. Anal. 25, no. 2, 417-452 (2015).

[12] J.A. Ball, V. Bolotnikov, De Branges-Rovnyak spaces: Basics and Theory, Operator Theory, Springer (2015), pp. 631-679.

[13] A. Baranov, E. Fricain, J. Mashreghi, Weighted norm inequalities for de Branges-Rovnyak spaces and their applications. Amer. J. Math. 132, no. 1, 125-155 (2010).

[14] T. Bermúdez, A. Martinón, J.A. Noda, An isometry plus a nilpotent operator is an m-isometry. Applications. J. Math. Anal. Appl. 407, no. 2, 505-512 (2013).

[15] A. Blandignères, E. Fricain, F. Gaunard, A. Hartmann, W. T. Ross, Direct and reverse Carleson measures for $H(b)$ spaces. Indiana Univ. Math. J. 64, no. 4, 1027-1057 (2015).

[16] N. Chevrot, D. Guillot, T. Ransford, De Branges-Rovnyak spaces and Dirichlet spaces. J. Funct. Anal. 259, no. 9, 2366-2383 (2010).

[17] C. Costara, T. Ransford, Which de Branges-Rovnyak spaces are Dirichlet spaces (and vice versa)? J. Funct. Anal. 265 (12), 3204-3218 (2013). 
[18] O. El-Fallah, E. Fricain, K. Kellay, J. Mashreghi, T. Ransford, Constructive approximation in de Branges-Rovnyak spaces. Constr. Approx. 44, no. 2, 269281 (2016).

[19] L. de Branges, J. Rovnyak, Square Summable Power Series. Holt, Rinehart and Winston, New York (1966).

[20] O. El-Fallah, K. Kellay, H. Klaja, J. Mashreghi, T. Ransford, Dirichlet spaces with superharmonic weights and de Branges-Rovnyak spaces. Complex Anal. Oper. Theory 10 (2016), no. 1, 97-107.

[21] E. Fricain, A. Hartmann, W. T. Ross, Concrete examples of $\mathcal{H}(b)$ spaces. Comput. Methods Funct. Theory 16, no. 2, 287-306 (2016).

[22] E. Fricain, M. Karaki, J. Mashreghi, Composition operators on de BrangesRovnyak spaces. Results Math. 74, no. 1, Art. 61, 18 pp (2019).

[23] E. Fricain, J. Mashreghi, The theory of $\mathcal{H}(b)$ spaces. Vols. 1 and 2, New Mathematical Monographs, 20 and 21, Cambridge University Press, Cambridge, 2016.

[24] Z.J. Jabłonski, I.B. Jung, J. Stochel, $m$-Isometric operators and their local properties. Linear Algebra Appl. 596, 49-70 (2020).

[25] C. Gu and S. Luo, Composition and multiplication operators on the derivative Hardy space $S^{2}(\mathbb{D})$. Complex Var. Elliptic Equ. 63 , no. 5, 599-624 (2018).

[26] H. Helton, Operators with a representation as multiplication by x on a Sobolev space. Hilbert space operators and operator algebras (Proc. Internat. Conf., Tihany, 1970), pp. 279-287. (loose errata) Colloq. Math. Soc. János Bolyai, 5, North-Holland, Amsterdam, 1972.

[27] K. Kellay, M. Zarrabi, Two-isometries and de Branges-Rovnyak spaces. Complex Anal. Oper. Theory 9, no. 6, 1325-1335 (2015).

[28] B. Łanucha, M. T. Nowak, Examples of de Branges-Rovnyak spaces generated by nonextreme functions. Ann. Acad. Sci. Fenn. Math. 44, no. 1, 449-457 (2019).

[29] S. Luo, S. Richter, Hankel operators and invariant subspaces of the Dirichlet space. J. Lond. Math. Soc. (2) 91 (2015), no. 2, 423-438.

[30] J. Mashreghi, D. Timotin, Nonextreme de Branges-Rovnyak spaces as models for contractions. Integral Equations Operator Theory 80, no. 1, 137-152 (2014).

[31] S. Richter, Invariant subspaces in Banach spaces of analytic functions, Trans. Amer. Math. Soc. 304, no. 2, 585-616 (1987).

[32] S. Richter, Invariant subspaces of the Dirichlet shift, J. Reine Angew. Math. 386, 205-220 (1988).

[33] S. Richter, A representation theorem for cyclic analytic two-isometries, Trans. Amer. Math. Soc. 328, 325-349(1991).

[34] S. Richter, C. Sundberg, A formula for the local Dirichlet integral, Michigan Math. J. 38, 355-379 (1991).

[35] F. Riesz, B. Sz.-Nagy, Functional analysis. Dover Books on Advanced Mathematics. Dover Publications, Inc., New York (1990).

[36] W. Rudin, Function theory in the unit ball of $\mathbb{C}^{n}$. Reprint of the 1980 edition. Classics in Mathematics. Springer-Verlag, Berlin, 2008.

[37] S. Shimorin, Wold-type decompositions and wandering subspaces for operators close to isometries. J. Reine Angew. Math. 531, 147-189 (2001).

[38] D. Sarason, Sub-Hardy Hilbert Spaces in the Unit Disk. Wiley, New York (1994). 
[39] D. Sarason, Local Dirichlet spaces as de Branges-Rovnyak spaces. Proc. Amer. Math. Soc. 125 (1997), no. 7, 2133-2139.

School of Mathematics, Hunan University, Changsha, Hunan, 410082, PR CHINA

E-mail address: sluo@hnu.edu.cn

Department of Mathematics, California Polytechnic State UniverSity, SAN Luis OBISPo, CA 93407, USA

E-mail address: cgu@calpoly.edu

Department of Mathematics, University of Tennessee, Knoxville, TN, 37996, USA

E-mail address: srichter@utk.edu 